\title{
Fourier Multipliers on Triebel-Lizorkin-Type Spaces
}

\section{Dachun Yang, Wen Yuan, and Ciqiang Zhuo}

School of Mathematical Sciences, Beijing Normal University, Laboratory of Mathematics and Complex Systems, Ministry of Education, Beijing 100875, China

Correspondence should be addressed to Wen Yuan, wenyuan@bnu.edu.cn

Received 20 November 2011; Accepted 10 January 2012

Academic Editor: Hans Triebel

Copyright (C) 2012 Dachun Yang et al. This is an open access article distributed under the Creative Commons Attribution License, which permits unrestricted use, distribution, and reproduction in any medium, provided the original work is properly cited.

\begin{abstract}
The authors study the mapping properties of Fourier multipliers, with symbols satisfying some generalized Hörmander's condition, on Triebel- Lizorkin-type spaces and Triebel-Lizorkin-Hausdorff spaces. To this end, the authors first establish a new characterization of these spaces via some generalized (weighted) $g_{\curlywedge}^{*}$ functions, which essentially improves the known result for TriebelLizorkin spaces even when $\tau=0$. Applying this new characterization, the authors then obtain the boundedness of Fourier multipliers on Triebel-Lizorkin-type spaces and Triebel-Lizorkin-Hausdorff spaces, which also give a new proof of the Sobolev embedding theorems for these spaces.
\end{abstract}

\section{Introduction}

It is well known that many classical operators, including some convolution operators, fractional differential operators, and pseudodifferential operators with constant coefficients, fall into the framework of Fourier multipliers. The study of mapping properties of Fourier multipliers on Besov and Triebel-Lizorkin spaces has a long history; see, for example, [1-10]. Indeed, the best-known Fourier multiplier on $L^{p}\left(\mathbb{R}^{n}\right)$ for $p \in(1, \infty)$, which is nowadays called Hörmander's multiplier theorem, was obtained by Hörmander [3, Theorem 2.5], preceded by Mihlin [1, 2]. Triebel [4, Theorem 3.5] gave a very useful generalization of Hörmander's multiplier theorem [3, Theorem 2.5] from the scalar-valued case to the vector-valued case, which further induced the introduction of the nowadays called Triebel-Lizorkin spaces; see also [5, pages 161-168] for more details including some history of the study on Fourier multipliers. Later, Triebel [9, Theorem 2] established a Fourier multiplier theorem for inhomogeneous Triebel-Lizorkin spaces, which was even proved to be sharp in [9, Remark 12]; see also [10, pages 73-77] for a detailed discussion. 
Recently, Cho and Kim [11] and Cho [12] introduced a new family of Fourier multipliers with symbols satisfying some generalized Hörmander's condition and studied the mapping properties of these Fourier multipliers on the classical homogeneous Besov spaces $\dot{B}_{p, q}^{s}\left(\mathbb{R}^{n}\right)$ and Triebel-Lizorkin spaces $\dot{F}_{p, q}^{s}\left(\mathbb{R}^{n}\right)$ via first establishing some equivalent characterizations of these spaces. This family of Fourier multipliers contains the classical Riesz potential operator $I_{\alpha}$ and the differential operator $\partial^{\alpha}$ as special cases. As an application, Cho and Kim [11] and Cho [12] presented a new proof of the Sobolev embedding theorems for Besov and Triebel-Lizorkin spaces.

The main purpose of this paper is to clarify the behaviors of these Fourier multipliers in $[11,12]$ on four new classes of function spaces: the Besov-type space $\dot{B}_{p, q}^{s, \tau}\left(\mathbb{R}^{n}\right)$, the TriebelLizorkin-type space $\dot{F}_{p, q}^{s, \tau}\left(\mathbb{R}^{n}\right)$, and their preduals, the Besov-Hausdorff space $B \dot{H}_{p, q}^{s, \tau}\left(\mathbb{R}^{n}\right)$ and the Triebel-Lizorkin-Hausdorff space $F \dot{H}_{p, q}^{s, \tau}\left(\mathbb{R}^{n}\right)$. These spaces were recently introduced and investigated in [13-18] and proved therein to cover many classical function spaces such as Besov spaces and Triebel-Lizorkin spaces (see, e.g., $[10,19,20]), Q$ spaces and Hardy-Hausdorff spaces (see, e.g., [21-24]), Triebel-Lizorkin-Morrey spaces and Morrey spaces (see, e.g., $[16,25-28])$. To study the boundedness of Fourier multipliers on $\dot{F}_{p, q}^{s, \tau}\left(\mathbb{R}^{n}\right)$ and $F \dot{H}_{p, q}^{s, \tau}\left(\mathbb{R}^{n}\right)$, we first establish a new characterization of these spaces in terms of generalized (weighted) $g_{\lambda}^{*}$ functions, which essentially improve the known results in [12] for Triebel-Lizorkin spaces even when $\tau=0$. Applying this new characterization, we then obtain the Fourier multiplier results on $\dot{F}_{p, q}^{s, \tau}\left(\mathbb{R}^{n}\right)$ and $F \dot{H}_{p, q}^{s, \tau}\left(\mathbb{R}^{n}\right)$, which also essentially improve the known results for Triebel-Lizorkin spaces obtained by Cho in [12] and, moreover, give a new proof of the Sobolev embedding theorems, obtained in $[14,15]$, for these spaces. Besides, for the Besovtype space $\dot{B}_{p, q}^{s, \tau}\left(\mathbb{R}^{n}\right)$ and the Besov-Hausdorff space $B \dot{H}_{p, q}^{s, \tau}\left(\mathbb{R}^{n}\right)$, some of the corresponding results are also presented.

We begin with some notions and notation. In what follows, let $\mathbb{N}:=\{1,2, \ldots\}$ and $\mathbb{Z}_{+}:=$ $\mathbb{N} \cup\{0\}$; let $\mathcal{S}\left(\mathbb{R}^{n}\right)$ be the space of all the Schwartz functions on $\mathbb{R}^{n}$ with the classical topology and $S^{\prime}\left(\mathbb{R}^{n}\right)$ its topological dual space, namely, the set of all continuous linear functionals on $\mathcal{S}\left(\mathbb{R}^{n}\right)$ endowed with the weak-* topology.

Following Triebel [10], let

$$
\mathcal{S}_{\infty}\left(\mathbb{R}^{n}\right):=\left\{\varphi \in \mathcal{S}\left(\mathbb{R}^{n}\right): \int_{\mathbb{R}^{n}} \varphi(x) x^{\gamma} d x=0 \forall \text { multi-indices } \gamma \in(\mathbb{N} \cup\{0\})^{n}\right\}
$$

and consider $\mathcal{S}_{\infty}\left(\mathbb{R}^{n}\right)$ as a subspace of $\mathcal{S}\left(\mathbb{R}^{n}\right)$, including the topology. Use $\mathcal{S}_{\infty}^{\prime}\left(\mathbb{R}^{n}\right)$ to denote the topological dual space of $S_{\infty}\left(\mathbb{R}^{n}\right)$, namely, the set of all continuous linear functionals on $\mathcal{S}_{\infty}\left(\mathbb{R}^{n}\right)$. We also endow $\mathcal{S}_{\infty}^{\prime}\left(\mathbb{R}^{n}\right)$ with the weak-* topology. Let $P\left(\mathbb{R}^{n}\right)$ be the set of all polynomials on $\mathbb{R}^{n}$. It is well known that $S_{\infty}^{\prime}\left(\mathbb{R}^{n}\right)=\mathcal{S}^{\prime}\left(\mathbb{R}^{n}\right) / D\left(\mathbb{R}^{n}\right)$ as topological spaces. Similarly, for any $N \in \mathbb{Z}_{+}$, the space $\mathcal{S}_{N}\left(\mathbb{R}^{n}\right)$ is defined to be the set of all Schwartz functions satisfying that $\int_{\mathbb{R}^{n}} \varphi(x) x^{\gamma} d x=0$ for all multi-indices $\gamma \in \mathbb{Z}_{+}^{n}$ with $|\gamma| \leq N$ and $S_{N}^{\prime}\left(\mathbb{R}^{n}\right)$ its topological dual space. We also let $\mathcal{S}_{-1}\left(\mathbb{R}^{n}\right):=\mathcal{S}\left(\mathbb{R}^{n}\right)$. As usual, $\hat{\phi}$ denotes the Fourier transform of an integrable function $\phi$ on $\mathbb{R}^{n}$, which is defined as $\widehat{\phi}(\xi):=\int_{\mathbb{R}^{n}} e^{-i \xi \cdot x} \phi(x) d x$ for all $\xi \in \mathbb{R}^{n}$.

The following notion of Fourier multipliers when $\alpha \neq 0$ was originally introduced by Cho and Kim in [11] and Cho in [12]. For $\ell \in \mathbb{N}$ and $\alpha \in \mathbb{R}$, assume that $m \in C^{\ell}\left(\mathbb{R}^{n} \backslash\{0\}\right)$ satisfies that for all $|\sigma| \leq \ell$,

$$
\sup _{R \in(0, \infty)}\left[R^{-n+2 \alpha+2|\sigma|} \int_{R \leq|\xi|<2 R}\left|\partial_{\xi}^{\sigma} m(\xi)\right|^{2} d \xi\right] \leq A_{\sigma}<\infty,
$$


where for $\sigma:=\left(\sigma_{1}, \ldots, \sigma_{n}\right) \in \mathbb{Z}_{+}^{n}, \partial^{\sigma}:=\left(\partial / \partial x_{1}\right)^{\sigma_{1}} \ldots\left(\partial / \partial x_{n}\right)^{\sigma_{n}}$. The Fourier multiplier $T_{m}$ is defined by setting, for all $f \in \mathcal{S}_{\infty}\left(\mathbb{R}^{n}\right), \widehat{\left(T_{m} f\right)}:=m \widehat{f}$.

We remark that the condition (1.2) when $\alpha=0$ is just the classical Hörmander condition (see [3, Theorem 2.5]) and, moreover, the condition (1.2) when $\alpha=0$ with maximum norms instead of $L^{2}$ norms is called the Mihlin condition (see [1,2]). One typical example satisfying (1.2) with $\alpha=0$ is the kernels of Riesz transforms $R_{j}$ given by

$$
\widehat{\left(R_{j} f\right)}(\xi):=-i\left(\xi_{i} /|\xi|\right) \widehat{f}(\xi)
$$

for $\xi \in \mathbb{R}^{n} \backslash\{0\}$ and $j \in\{1, \ldots, n\}$. When $\alpha \neq 0$, a typical example satisfying (1.2) for any $\ell \in \mathbb{N}$ is given by

$$
m(\xi):=|\xi|^{-\alpha} \text { for } \xi \in \mathbb{R}^{n} \backslash\{0\},
$$

another example is the symbol of a differential operator $\partial^{\sigma}$ of order $\alpha:=\sigma_{1}+\cdots+\sigma_{n}$ with $\sigma:=\left(\sigma_{1}, \ldots, \sigma_{n}\right) \in \mathbb{Z}_{+}^{n}$.

To recall the notions of $\dot{B}_{p, q}^{s, \tau}\left(\mathbb{R}^{n}\right)$ and $\dot{F}_{p, q}^{s, \tau}\left(\mathbb{R}^{n}\right)$ in [14] and, their predual spaces, $B \dot{H}_{p, q}^{s, \tau}\left(\mathbb{R}^{n}\right)$ and $F \dot{H}_{p, q}^{s, \tau}\left(\mathbb{R}^{n}\right)$ in $[13,14]$, we need the following notation.

For $j \in \mathbb{Z}$ and $k \in \mathbb{Z}^{n}$, denote by $Q_{j k}$ the dyadic cube $2^{-j}\left([0,1)^{n}+k\right)$ and $\ell\left(Q_{j k}\right)$ its side length. Let $Q:=\left\{Q_{j k}: j \in \mathbb{Z}, k \in \mathbb{Z}^{n}\right\}, Q_{j}:=\left\{Q \in Q: \ell(Q)=2^{-j}\right\}$ and $j_{Q}:=-\log _{2} \ell(Q)$ for all $Q \in Q$.

Let $q \in(0, \infty]$ and $\tau \in[0, \infty)$. The space $L_{\tau}^{p}\left(\ell^{q}\left(\mathbb{R}^{n}, \mathbb{Z}\right)\right)$ with $p \in(0, \infty)$ is defined to be the set of all sequences $G:=\left\{g_{j}\right\}_{j \in \mathbb{Z}}$ of measurable functions on $\mathbb{R}^{n}$ such that

$$
\|G\|_{L_{\tau}^{p}\left(\mathcal{Q q}^{q}\left(\mathbb{R}^{n}, \mathbb{Z}\right)\right)}:=\sup _{P \in \mathcal{Q}} \frac{1}{|P|^{\tau}}\left\{\int_{P}\left[\sum_{j=j_{P}}^{\infty}\left|g_{j}(x)\right|^{q}\right]^{p / q} d x\right\}^{1 / p}<\infty .
$$

Similarly, the space $\ell^{q}\left(L_{\tau}^{p}\left(\mathbb{R}^{n}, \mathbb{Z}\right)\right)$ with $p \in(0, \infty]$ is defined to be the space of all sequences $G:=\left\{g_{j}\right\}_{j \in \mathbb{Z}}$ of measurable functions on $\mathbb{R}^{n}$ such that

$$
\|G\|_{\ell q\left(L_{\tau}^{p}\left(\mathbb{R}^{n}, \mathbb{Z}\right)\right)}:=\sup _{P \in \mathcal{Q}} \frac{1}{|P|^{\tau}}\left\{\sum_{j=j_{P}}^{\infty}\left[\int_{P}\left|g_{j}(x)\right|^{p} d x\right]^{q / p}\right\}^{1 / q}<\infty .
$$

Throughout the whole paper, we denote by $\mathrm{C}$ a positive constant which is independent of the main parameters, but it may vary from line to line. Let $\mathcal{A}$ be the space of all functions $\varphi \in$ $\mathcal{S}\left(\mathbb{R}^{n}\right)$ such that

$$
\operatorname{supp} \widehat{\varphi} \subset\left\{\xi \in \mathbb{R}^{n}: \frac{1}{2} \leq|\xi| \leq 2\right\}, \quad|\widehat{\varphi}(\xi)| \geq C>0 \quad \text { if } \frac{3}{5} \leq|\xi| \leq \frac{5}{3}
$$

Now we recall the notions of the Besov-type space $\dot{B}_{p, q}^{s, \tau}\left(\mathbb{R}^{n}\right)$ and the Triebel-Lizorkin-type space $\dot{F}_{p, q}^{s, \tau}\left(\mathbb{R}^{n}\right)$ from [14]. In what follows, for any $j \in \mathbb{Z}$ and $\varphi \in \mathcal{A}$, let $\varphi_{j}(x):=2^{j n} \varphi\left(2^{j} x\right)$ for all $x \in \mathbb{R}^{n}$. 
Definition 1.1. Let $s \in \mathbb{R}, \tau \in[0, \infty), q \in(0, \infty]$ and $\varphi \in \mathcal{A}$.

(i) The Besov-type space $\dot{B}_{p, q}^{s, \tau}\left(\mathbb{R}^{n}\right)$ with $p \in(0, \infty]$ is defined to be the space of all $f \in$ $S_{\infty}^{\prime}\left(\mathbb{R}^{n}\right)$ such that $\|f\|_{\dot{B}_{p, q}^{s, \tau}\left(\mathbb{R}^{n}\right)}:=\left\|\left\{2^{j s}\left(\varphi_{j} * f\right)\right\}_{j \in \mathbb{Z}}\right\|_{\ell^{q}\left(L_{\tau}^{p}\left(\mathbb{R}^{n}, \mathbb{Z}\right)\right)}<\infty$.

(ii) The Triebel-Lizorkin-type space $\dot{F}_{p, q}^{s, \tau}\left(\mathbb{R}^{n}\right)$ with $p \in(0, \infty)$ is defined to be the space of all $f \in S_{\infty}^{\prime}\left(\mathbb{R}^{n}\right)$ such that $\|f\|_{\dot{F}_{p, q}^{s, \tau}\left(\mathbb{R}^{n}\right)}:=\left\|\left\{2^{j s}\left(\varphi_{j} * f\right)\right\}_{j \in \mathbb{Z}}\right\|_{L_{\tau}^{p}\left(\ell q\left(\mathbb{R}^{n}, \mathbb{Z}\right)\right)}<\infty$.

Obviously, $\dot{B}_{p, q}^{s, 0}\left(\mathbb{R}^{n}\right)=\dot{B}_{p, q}^{s}\left(\mathbb{R}^{n}\right)$ and $\dot{F}_{p, q}^{s, 0}\left(\mathbb{R}^{n}\right)=\dot{F}_{p, q}^{s}\left(\mathbb{R}^{n}\right)$. We also remark that the spaces $\dot{B}_{p, q}^{s, \tau}\left(\mathbb{R}^{n}\right)$ and $\dot{F}_{p, q}^{s, \tau}\left(\mathbb{R}^{n}\right)$ are independent of the choice of $\varphi \in \mathscr{A}$; see [14].

Remark 1.2. Let $s \in \mathbb{R}$.

(i) For $p \in(0, \infty)$, it was proved in [29, Theorem 1(i)] that $\dot{F}_{p, r}^{s, \tau}\left(\mathbb{R}^{n}\right)=\dot{F}_{\infty, \infty}^{s+n(\tau-1 / p)}\left(\mathbb{R}^{n}\right)$ when $r \in(0, \infty)$ and $\tau \in(1 / p, \infty)$, and $\dot{F}_{p, \infty}^{s, \tau}\left(\mathbb{R}^{n}\right)=\dot{F}_{\infty, \infty}^{s+n(\tau-1 / p)}\left(\mathbb{R}^{n}\right)$ when $\tau \in$ $[1 / p, \infty)$ with equivalent quasinorms. In [30, Corollary 5.7], it was proved that $\dot{F}_{p, q}^{s, 1 / p}\left(\mathbb{R}^{n}\right)=\dot{F}_{\infty, q}^{s}\left(\mathbb{R}^{n}\right)$ with equivalent quasinorms for $p \in(0, \infty)$ and $q \in(0, \infty]$.

(ii) For $\mathrm{p} \in(0, \infty]$, it was proved in $\left[29\right.$, Theorem 1 (ii)] that $\dot{B}_{p, r}^{s, \tau}\left(\mathbb{R}^{n}\right)=\dot{B}_{\infty, \infty}^{s+n(\tau-1 / p)}\left(\mathbb{R}^{n}\right)$ when $r \in(0, \infty)$ and $\tau \in(1 / p, \infty)$, and $\dot{B}_{p, \infty}^{s, \tau}\left(\mathbb{R}^{n}\right)=\dot{B}_{\infty, \infty}^{s+n(\tau-1 / p)}\left(\mathbb{R}^{n}\right)$ when $\tau \in$ $[1 / p, \infty)$ with equivalent quasinorms.

Next we recall the Hausdorff-type counterparts of $L_{\tau}^{p}\left(\ell^{q}\left(\mathbb{R}^{n}, \mathbb{Z}\right)\right)$ and $\ell^{q}\left(L_{\tau}^{p}\left(\mathbb{R}^{n}, \mathbb{Z}\right)\right)$. To this end, for $x \in \mathbb{R}^{n}$ and $r \in(0, \infty)$, let $B(x, r):=\left\{y \in \mathbb{R}^{n}:|x-y|<r\right\}$. For $E \subset \mathbb{R}^{n}$ and $d \in(0, n]$, the $d$-dimensional Hausdorff capacity of $E$ is defined by

$$
H^{d}(E):=\inf \left\{\sum_{j} r_{j}^{d}: E \subset \bigcup_{j} B\left(x_{j}, r_{j}\right)\right\}
$$

where the infimum is taken over all countable open ball coverings $\left\{B\left(x_{j}, r_{j}\right)\right\}_{j}$ of $E$; see, for example, [31, 32]. defined by

For any function $f: \mathbb{R}^{n} \rightarrow[0, \infty]$, the Choquet integral of $f$ with respect to $H^{d}$ is then

$$
\int_{\mathbb{R}^{n}} f(x) d H^{d}(x):=\int_{0}^{\infty} H^{d}\left(\left\{x \in \mathbb{R}^{n}: f(x)>\lambda\right\}\right) d \lambda
$$

In what follows, we write $\mathbb{R}_{+}^{n+1}:=\mathbb{R}^{n} \times(0, \infty)$. For any measurable function $\omega$ on $\mathbb{R}_{+}^{n+1}$ and $x \in \mathbb{R}^{n}$, its nontangential maximal function $N \omega$ is defined by

$$
N \omega(x):=\sup _{|y-x|<t}|\omega(y, t)|, \quad x \in \mathbb{R}^{n}
$$


For $p \in(1, \infty)$ and $\tau \in[0, \infty)$, the space $\left.L_{\tau}^{p}\left(\widetilde{\ell^{q}\left(\mathbb{R}^{n}\right.}, \mathbb{Z}\right)\right)$ with $q \in(1, \infty)$ is defined to be the space of all sequences $G:=\left\{g_{j}\right\}_{j \in \mathbb{Z}}$ of measurable functions on $\mathbb{R}^{n}$ such that

$$
\|G\|_{L_{\tau}^{p}\left(\widetilde{\left.\mathcal{R}^{q}\left(\mathbb{R}^{n}, \mathbb{Z}\right)\right)}\right.}:=\inf _{\omega}\left\{\int_{\mathbb{R}^{n}}\left(\sum_{j \in \mathbb{Z}}\left|g_{j}(x)\right|^{q}\left[\omega\left(x, 2^{-j}\right)\right]^{-q}\right)^{p / q} d x\right\}^{1 / q}<\infty,
$$

and the space $\left.\ell^{q}\left(\widetilde{L_{\tau}^{p}\left(\mathbb{R}^{n}\right.}, \mathbb{Z}\right)\right)$ with $q \in[1, \infty)$ is defined to be the space of all sequences $G:=$ $\left\{g_{j}\right\}_{j \in \mathbb{Z}}$ of measurable functions on $\mathbb{R}^{n}$ such that

$$
\|G\|_{\ell q\left(\widetilde{\left.L_{\tau}^{p}\left(\mathbb{R}^{n}, \mathbb{Z}\right)\right)}\right.}:=\inf _{\omega}\left\{\sum_{j \in \mathbb{Z}}\left(\int_{\mathbb{R}^{n}}\left|g_{j}(x)\right|^{p}\left[\omega\left(x, 2^{-j}\right)\right]^{-p} d x\right)^{q / p}\right\}^{1 / p}<\infty,
$$

where the infimums are taken over all nonnegative Borel measurable functions $\omega$ on $\mathbb{R}_{+}^{n+1}$ satisfying

$$
\int_{\mathbb{R}^{n}}[N \omega(x)]^{(p \vee q) \prime} d H^{n \tau(p \vee q) \prime}(x) \leq 1,
$$

and with the restriction that for any $j \in \mathbb{Z}, \omega\left(\cdot, 2^{-j}\right)$ is allowed to vanish only where $g_{j}$ vanishes. Here and, in what follows, for all $a, b \in \mathbb{R}$, the symbol $a \vee b$ denotes $\max \{a, b\}$ and, for $t \in[1, \infty]$, the symbol $t^{\prime}$ denotes its conjugate index, namely, $1 / t+1 / t^{\prime}=1$.

Remark 1.3. By [15, Remark 2.1], we know that if $0<a \leq b \leq 1 / \tau$, then for all nonnegative measurable functions $\omega$ on $\mathbb{R}_{+}^{n+1}$,

$$
\int_{\mathbb{R}^{n}}[N \omega(x)]^{a} d \mathrm{H}^{n \tau a}(x) \leq 1 \quad \text { implies that } \int_{\mathbb{R}^{n}}[N \omega(x)]^{b} d H^{n \tau b}(x) \lesssim 1
$$

We now recall the notion of the spaces $B \dot{H}_{p, q}^{s, \tau}\left(\mathbb{R}^{n}\right)$ and $F \dot{H}_{p, q}^{s, \tau}\left(\mathbb{R}^{n}\right)$ introduced in [17].

Definition 1.4. Let $s \in \mathbb{R}, p \in(1, \infty)$ and $\varphi \in \mathcal{A}$.

(i) The Besov-Hausdorffspace $B \dot{H}_{p, q}^{s, \tau}\left(\mathbb{R}^{n}\right)$ with $q \in[1, \infty)$ and $\tau \in\left[0,1 /(p \vee q)^{\prime}\right]$ is defined to be the space of all $f \in S_{\infty}^{\prime}\left(\mathbb{R}^{n}\right)$ such that

$$
\|f\|_{B \dot{H}_{p, q}^{s, \tau}\left(\mathbb{R}^{n}\right)}:=\left\|\left\{2^{j s}\left(\varphi_{j} * f\right)\right\}_{j \in \mathbb{Z}}\right\|_{\mathcal{\ell}^{q}\left(L_{\tau}^{p}\left(\mathbb{R}^{n}, \mathbb{Z}\right)\right)}<\infty .
$$


(ii) The Triebel-Lizorkin-Hausdorff space $F \dot{H}_{p, q}^{s, \tau}\left(\mathbb{R}^{n}\right)$ with $q \in(1, \infty)$ and $\tau \in\left[0,1 /(p \vee q)^{\prime}\right]$ is defined to be the space of all $f \in S_{\infty}^{\prime}\left(\mathbb{R}^{n}\right)$ such that

$$
\|f\|_{F \dot{H}_{p, q}^{s, \tau}\left(\mathbb{R}^{n}\right)}:=\left\|\left\{2^{j s}\left(\varphi_{j} * f\right)\right\}_{j \in \mathbb{Z}}\right\|_{L_{\tau}^{p}\left(\widetilde{\left.\ell q\left(\mathbb{R}^{n}, \mathbb{Z}\right)\right)}\right.}<\infty
$$

Recall that $B \dot{H}_{p, q}^{s, 0}\left(\mathbb{R}^{n}\right)=\dot{B}_{p, q}^{s}\left(\mathbb{R}^{n}\right)$ and $F \dot{H}_{p, q}^{s, 0}\left(\mathbb{R}^{n}\right)=\dot{F}_{p, q}^{s}\left(\mathbb{R}^{n}\right)$. Moreover, the dual spaces of $B \dot{H}_{p, q}^{s, \tau}\left(\mathbb{R}^{n}\right)$ and $F \dot{H}_{p, q}^{s, \tau}\left(\mathbb{R}^{n}\right)$ are, respectively, $\dot{B}_{p^{\prime}, q^{\prime}}^{-s, \tau}\left(\mathbb{R}^{n}\right)$ and $\dot{F}_{p^{\prime}, q^{\prime}}^{-s, \tau}\left(\mathbb{R}^{n}\right)$; see $[13,14]$.

Now we present the main results of this paper as follows.

Theorem 1.5. Let $\alpha, \gamma \in \mathbb{R}, \tau \in[0, \infty)$, and $r \in(0, \infty]$. Suppose that $m$ satisfies (1.2) with $\ell \in \mathbb{N}$.

(i) If $\ell>n[\max (1 / p, 1 / r)+1 / 2]$ and $p \in(0, \infty)$, then there exists a positive constant $C$ such that for all $f \in \dot{F}_{p, r}^{\gamma, \tau}\left(\mathbb{R}^{n}\right),\left\|T_{m} f\right\|_{\dot{F}_{p, r}^{\alpha+r, \tau}\left(\mathbb{R}^{n}\right)} \leq C\|f\|_{\dot{F}_{p, r}^{r, \tau}\left(\mathbb{R}^{n}\right)}$.

(ii) If $\ell>n(1 / p+1 / 2)$ and $p \in(0, \infty]$, then there exists a positive constant $C$ such that for all $f \in \dot{B}_{p, r}^{\gamma, \tau}\left(\mathbb{R}^{n}\right),\left\|T_{m} f\right\|_{\dot{B}_{p, r}^{\alpha+\gamma}\left(\mathbb{R}^{n}\right)} \leq C\|f\|_{\mathcal{B}_{p, r}^{\gamma, \tau}\left(\mathbb{R}^{n}\right)}$.

We remark that the Fourier multiplier $T_{m}$ is originally defined on $\mathcal{S}_{\infty}\left(\mathbb{R}^{n}\right)$. Although $\mathcal{S}_{\infty}\left(\mathbb{R}^{n}\right)$ may not be dense in $\dot{F}_{p, q}^{s, \tau}\left(\mathbb{R}^{n}\right)$ and $\dot{B}_{p, q}^{s, \tau}\left(\mathbb{R}^{n}\right), T_{m}$ can still be defined on the whole spaces $\dot{F}_{p, q}^{s, \tau}\left(\mathbb{R}^{n}\right)$ and $\dot{F}_{p, q}^{s, \tau}\left(\mathbb{R}^{n}\right)$ in a suitable way; see (3.10) and Lemma 3.4 below.

We also remark Theorem 1.5 when $\tau=0$ completely covers the known results obtained in [12, Theorem 5.1]. The proof of Theorem 1.5 is given in Section 3.

From Theorem 1.5 and [14, Proposition 3.3], we immediately deduce the following conclusion. We omit the details.

Corollary 1.6. Let $\alpha, \beta \in \mathbb{R}, \beta<\alpha, p \in(0, \infty), q, r \in(0, \infty]$, and $\tau \in[0, \infty)$. Assume that $m$ satisfies (1.2) with $\ell \in \mathbb{N}$.

(i) If $\ell>n[\max (1 / p, 1 / r)+1 / 2]$ and $p_{*} \in(0, \infty)$ such that $\beta-n / p_{*}=\alpha-n / p$, then there exists a positive constant $C$ such that for all $f \in \dot{F}_{p, r}^{0, \tau}\left(\mathbb{R}^{n}\right)$,

$$
\left\|T_{m} f\right\|_{{\dot{\dot{F}_{*}, q}}_{\beta, \tau}^{\beta,\left(\mathbb{R}^{n}\right)}} \leq C\|f\|_{\dot{F}_{p, r}^{0, \tau}\left(\mathbb{R}^{n}\right)}
$$

(ii) If $\ell>n(1 / p+1 / 2)$ and $p_{*} \in(0, \infty]$ such that $\beta-n / p_{*}=\alpha-n / p$, then there exists a positive constant $C$ such that for all $f \in \dot{B}_{p, r}^{0, \tau}\left(\mathbb{R}^{n}\right)$,

$$
\left\|T_{m} f\right\|_{{\dot{B_{p}, r}}_{p, r}^{\beta, \tau}\left(\mathbb{R}^{n}\right)} \leq C\|f\|_{\dot{B}_{p, r}^{0, \tau}\left(\mathbb{R}^{n}\right)}
$$

We point out that Corollary 1.6(ii) when $\tau=0$ completely covers Cho and Kim [11, Theorem 1.1] and Cho [12, Theorem 7.1].

Moreover, the range of $\ell$ in Corollary 1.6(i) can be essentially improved as indicated by the following theorem. 
Theorem 1.7. Let $\alpha, \beta \in \mathbb{R}, p \in(0, \infty), \tau \in[0, \infty)$ and $r, q \in(0, \infty]$ such that $\beta<\alpha$. Let $p_{*} \in$ $(0, \infty)$ such that $\beta-n / p_{*}=\alpha-n / p$. Assume that $m$ satisfies (1.2) with $\ell \in \mathbb{N}$ and $\ell>n / 2$. Then there exists a positive constant $C$ such that for all $f \in \dot{F}_{p, r}^{0, \tau}\left(\mathbb{R}^{n}\right)$,

$$
\left\|T_{m} f\right\|_{\dot{F}_{p *, q}^{\beta, \tau},\left(\mathbb{R}^{n}\right)} \leq C\|f\|_{\dot{F}_{p, r}^{0, \tau}\left(\mathbb{R}^{n}\right)} .
$$

As an immediate consequence of Theorem 1.7 and the lifting property of the space $\dot{F}_{p, q}^{s, \tau}\left(\mathbb{R}^{n}\right)$ (see, [14, Proposition 3.5]), we have the following conclusion, which shows that Theorem 1.7 has variant for any $s \in \mathbb{R}$ instead of $s=0$.

Corollary 1.8. Given $\alpha, \gamma \in \mathbb{R}, p \in(0, \infty)$ and $r, q \in(0, \infty]$, let $\beta$ be real number with $\beta<\alpha+\gamma$ and $p_{*} \in(0, \infty)$ such that $\beta-n / p_{*}=\alpha+\gamma-n / p$. Assume that $m$ satisfies (1.2) with $\ell \in \mathbb{N}$ and $\ell>n / 2$. Then there exists a positive constant $C$ such that for all $f \in \dot{F}_{p, r}^{\gamma, \tau}\left(\mathbb{R}^{n}\right),\left\|T_{m} f\right\|_{\dot{F}_{p * q}^{\beta, \tau}\left(\mathbb{R}^{n}\right)} \leq C\|f\|_{\dot{F}_{p, r}^{\gamma, \tau}\left(\mathbb{R}^{n}\right)}$.

Remark 1.9. (i) We remark that, by taking $\beta=0, \alpha \in(0, n), p \in(1, n / \alpha), q=r=2, \tau=0$, and $m(\xi):=|\xi|^{-\alpha}$ for all $\xi \in \mathbb{R}^{n} \backslash\{0\}$, then Theorem 1.7 (and also Corollary 1.8 with $\gamma=0$ ) is just the well-known Hardy-Littlewood-Sobolev theorem for fractional integrals (see, e.g., [33, page 119 , Theorem $1(\mathrm{~b})]$ ), namely, the Riesz potential $I_{\alpha}$ maps boundedly from $L^{p}\left(\mathbb{R}^{n}\right)$ to $L^{p_{*}}\left(\mathbb{R}^{n}\right)$, where $1 / p_{*}=1 / p-\alpha / n$. In this sense, Theorem 1.7 (and hence Corollary 1.8) is a generalization of the Hardy-Littlewood-Sobolev for fractional integrals.

(ii) Theorem 1.7 (resp., Corollary 1.8) is not true in the case that $\beta=\alpha$ and hence $p_{*}=p$ (resp., $\beta=\gamma+\alpha$ and hence $p_{*}=p$ ). Indeed, the assumption $\beta<\alpha$ (resp., $\beta<\gamma+\alpha$ ) and hence $p_{*}>p$ play a crucial role in the proof of Theorem 1.7 in Section 3, which is not valid for the case that $\beta=\alpha$ (resp., $\beta=\gamma+\alpha$ ) and hence $p_{*}=p$.

For $\ell \in(0, \infty)$, let $W_{2}^{\ell}\left(\mathbb{R}^{n}\right)$ be the well-known Sobolev-Slobodeckij space on $\mathbb{R}^{n}$. Recall that Triebel $[9$, Theorem 2] proved that for all $s \in \mathbb{R}, p, q \in(0, \infty)$ and $\ell>n(1 / \min \{p, q, 1\}-1 / 2)$, if $m \in L^{\infty}\left(\mathbb{R}^{n}\right)$ and

$$
\|\psi m\|_{W_{2}^{e}\left(\mathbb{R}^{n}\right)}+\sup _{j \in \mathbb{N}}\left\|\varphi(\cdot) m\left(2^{j} \cdot\right)\right\|_{W_{2}^{e}\left(\mathbb{R}^{n}\right)}<\infty
$$

then the Fourier multiplier $T_{m}$ is bounded from the inhomogeneous Triebel-Lizorkin space $F_{p, q}^{s}\left(\mathbb{R}^{n}\right)$ to itself, where $\psi$ and $\varphi$ are Schwartz functions satisfying that $0 \leq \psi, \varphi \leq 1$, supp $\psi \subset \overline{B(0,2)}$, $\psi \equiv 1$ on $\overline{B(0,1)}$, supp $\varphi \subset \overline{B(0,4)} \backslash B(0,1 / 2)$ and $\varphi \equiv 1$ on $\overline{B(0,2)} \backslash B(0,1)$. From this, together with the embedding theorem [10, Theorem 2.7.1], we further deduce that, under the above assumptions on $m, T_{m}$ is also bounded from $F_{p, q}^{s}\left(\mathbb{R}^{n}\right)$ to $F_{p_{*}, r}^{s+n / p_{*}-n / p}\left(\mathbb{R}^{n}\right)$ with $s \in \mathbb{R}, p, q \in(0, \infty)$, $p_{*} \in(p, \infty)$ and $r \in(0, \infty]$.

Notice that, if $m$ is as in Theorem 1.7 or Corollary 1.8, then $m$ is not necessary to belong to $L^{\infty}\left(\mathbb{R}^{n}\right)$. For example, if $\alpha \neq 0$, then $m$ as in (1.4) satisfies all the assumptions of Theorem 1.7 and Corollary 1.8, but $m \notin L^{\infty}\left(\mathbb{R}^{n}\right)$. Thus, the assumptions in both Theorem 1.7 (or Corollary 1.8) and Triebel [9, Theorem 2] are not comparable. This is quite natural, since we are considering the multiplier on homogeneous function spaces, while Triebel $[9$, Theorem 2] (see also [10, pages 73-77]) studied the multipliers on inhomogeneous function spaces. In some sense, Theorem 1.7 and Corollary 1.8 might be regarded as fractional variants of the homogeneous version of [9, Theorem 2] which corresponds to the case that $\alpha=0$ of Theorem 1.7 and Corollary 1.8. This might also be the reason why the assumption on $\ell$ in $[9$, Theorem 2] is quite different from the requirement of $\ell$ in Theorem 1.7 and Corollary 1.8. Moreover, 
the restriction $\ell>n(1 / \min \{p, q, 1\}-1 / 2)$ in [9, Theorem 2] is sharp; see Triebel $[9$, Remark 12] or [10, pages 73-77].

(iii) Recall that in [34], Marschall introduced a very general class $S B_{\delta}^{k}(r, \mu, v ; N, \lambda)$ of symbols $a \in \mathcal{S}^{\prime}\left(\mathbb{R}^{n} \times \mathbb{R}^{n}\right)$ with $k \in \mathbb{R}, \delta \in[0,1], \mu, v \in(0, \infty], r \in[n / \mu, \infty) \cap(0, \infty), \lambda \in[1, \infty]$ and $N \in(n / \lambda, \infty)$. For a symbol $a \in S B_{\delta}^{k}(r, \mu, v ; N, \lambda)$, and any $f \in \mathcal{S}^{\prime}\left(\mathbb{R}^{n}\right)$ and $x \in \mathbb{R}^{n}$, the nonregular pseudodifferential operator $a(x, D)$ is defined as

$$
a(x, D) f(x):=\frac{1}{(2 \pi)^{n}} \int_{\mathbb{R}^{n}} e^{i x \cdot \xi} a(x, \xi) f(\xi) d \xi .
$$

Then Marschall [34, Theorem 9(a)] proved that for all $k \in \mathbb{R}, p, q \in(0, \infty], \delta \in[0,1], \mu, v \in$ $(0, \infty], r \in(n /(1-\delta) \mu, \infty), \lambda \in[1, \infty], N \in(n \max \{1 / \lambda, 1 / 2,1 / p, 1 / q\}, \infty)$ and

$$
n\left(\max \left\{1, \frac{1}{p}+\frac{1}{\mu}\right\}-1\right)-(1-\delta) r=s<r-n \max \left\{\frac{1}{\mu}-\frac{1}{p}, 0\right\},
$$

if either $p \in(0,1](p \in(0,1)$ in case that $\mu=\infty)$ or $p \in(\mu, \infty] \cap[v, \infty]$, then the operator $a(x, D)$ with $a \in S B_{\delta}^{k}(r, \mu, v ; N, \lambda)$ is bounded from $F_{p, q}^{s+k}\left(\mathbb{R}^{n}\right)$ to $F_{p, q}^{s}\left(\mathbb{R}^{n}\right)$, where $F_{p, q}^{s}\left(\mathbb{R}^{n}\right)$ denotes the inhomogeneous Triebel-Lizorkin space. This, together with the Sobolev embedding properties of Triebel-Lizorkin spaces, further implies that the operator $a(x, D)$ is bounded from $F_{p, q}^{s+k}\left(\mathbb{R}^{n}\right)$ to $F_{p_{*}, t}^{s+n / p_{*}-n / p}\left(\mathbb{R}^{n}\right)$ with $p_{*} \in(p, \infty)$ and $t \in(0, \infty]$.

Notice that, if $m$ satisfies the assumptions of Theorem 1.7 or Corollary 1.8, then $m$ is not necessary to belong to $\mathcal{S}^{\prime}\left(\mathbb{R}^{n}\right)$; see, for example, $m$ as in (1.4) with $\alpha \in(0, \infty)$. Thus, by the same reason as in (ii), the assumptions in both Theorem 1.7 (or Corollary 1.8) and Marschall [34, Theorem 9(a)] are not comparable.

(iv) Recall that it was proved by Cho in [12, Theorem 5.2] that when $\ell>$ $n[\max (1 / p, 1 / 2)]$ if $r \in(0,2)$, or $\ell>n[\max (1 / p, 1 / r)+1 / 2-1 / r]$ if $r \in[2, \infty]$, the operator $T_{m}$ maps $\dot{F}_{p, r}^{0}\left(\mathbb{R}^{n}\right)$ boundedly into $\dot{F}_{p_{*}, q}^{\beta}\left(\mathbb{R}^{n}\right)$. However, from Theorem 1.7 , we deduce that this conclusion is also true when $\ell>n / 2$ if $r \in(0, \infty]$. Therefore, even when $\tau=0$, Theorem 1.7 also essentially improves [12, Theorem 5.2]. Moreover, there exists a gap in the proof of [12, Theorem 5.2] in the endpoint case when $p_{*}=\infty$, namely, the formula $[12,(5.6)]$ seems not enough for the first inequality in [12, page 853]. The proof of Theorem 1.7 seals this gap and is given in Section 3.

Theorems 1.5 and 1.7 have the following counterparts for Hausdorff-type spaces.

Theorem 1.10. Let $\alpha, \gamma \in \mathbb{R}, p \in(1, \infty)$ and $m$ satisfy (1.2) with $\ell \in \mathbb{N}$.

(i) If $r \in(1, \infty), \tau \in\left[0,1 /(p \vee r)^{\prime}\right]$ and $\ell>n[\max (1 / p, 1 / r)+\tau+1 / 2]$, then there exists a positive constant $C$ such that for all $f \in F \dot{H}_{p, r}^{\gamma, \tau}\left(\mathbb{R}^{n}\right)$,

$$
\left\|T_{m} f\right\|_{F \dot{H}_{p, r}^{\alpha, \gamma, \tau}\left(\mathbb{R}^{n}\right)} \leq C\|f\|_{F \dot{H}_{p, r}^{\gamma, \tau}\left(\mathbb{R}^{n}\right)} .
$$

(ii) If $r \in[1, \infty), \tau \in\left[0,1 /(p \vee r)^{\prime}\right]$ and $\ell>n(1 / p+\tau+1 / 2)$, then there exists a positive constant $C$ such that for all $f \in B \dot{H}_{p, r}^{\gamma, \tau}\left(\mathbb{R}^{n}\right)$,

$$
\left\|T_{m} f\right\|_{B \dot{H}_{p, r}^{\alpha+r}\left(\mathbb{R}^{n)}\right.} \leq C\|f\|_{B \dot{H}_{p, r}^{\gamma, \tau}\left(\mathbb{R}^{n}\right)} .
$$


Differently from the spaces $\dot{F}_{p, q}^{s, \tau}\left(\mathbb{R}^{n}\right)$ and $\dot{B}_{p, q}^{s, \tau}\left(\mathbb{R}^{n}\right)$, it is known that $S_{\infty}\left(\mathbb{R}^{n}\right)$ is dense in the spaces $F \dot{H}_{p, q}^{s, \tau}\left(\mathbb{R}^{n}\right)$ and $B \dot{H}_{p, q}^{s, \tau}\left(\mathbb{R}^{n}\right)$; see [13, Lemma 5.3] and [14, Lemma 6.3]. Thus, although $T_{m} f$ is originally defined on $\mathcal{S}_{\infty}\left(\mathbb{R}^{n}\right)$, we can extend $T_{m}$ into the whole spaces $F \dot{H}_{p, q}^{s, \tau}\left(\mathbb{R}^{n}\right)$ and $B \dot{H}_{p, q}^{s, \tau}\left(\mathbb{R}^{n}\right)$ by a density argument.

We remark that Theorem 1.10(i) when $\tau=0$ coincides with [12, Theorem 5.1] in the case that $p \in(0, \infty)$. The proof of Theorem 1.10 is also given in Section 3 .

From Theorem 1.10 and [15, Theorem 4.1], we immediately deduce the following conclusion and omit the details.

Corollary 1.11. Let $\alpha, \beta \in \mathbb{R}, \beta<\alpha, p \in(1, \infty)$ and $p_{*} \in(1, \infty)$ such that $\beta-n / p_{*}=\alpha-n / p$. Assume that $m$ satisfies the condition (1.2) with $\ell \in \mathbb{N}$.

(i) Let $r, q \in(1, \infty)$ and $\tau \in\left[0, \min \left\{1 /(p \vee r)^{\prime}, 1 /\left(p_{*} \vee q\right)^{\prime}\right\}\right]$ such that $\tau(p \vee r)^{\prime} \leq \tau\left(p_{*} \vee q\right)^{\prime}$. If $\ell>n[\max (1 / p, 1 / r)+\tau+1 / 2]$, then there exists a positive constant $C$ such that for all $f \in F \dot{H}_{p, r}^{0, \tau}\left(\mathbb{R}^{n}\right)$,

$$
\left\|T_{m} f\right\|_{F \dot{H}_{p *, q}^{\beta, \tau}\left(\mathbb{R}^{n}\right)} \leq C\|f\|_{F \dot{H}_{, r}^{0, \tau}\left(\mathbb{R}^{n}\right)}
$$

(ii) Let $r \in[1, \infty)$ and $\tau \in\left[0, \min \left\{1 /(p \vee r)^{\prime}, 1 /\left(p_{*} \vee r\right)^{\prime}\right\}\right]$ such that $\tau(p \vee r)^{\prime}=\tau\left(p_{*} \vee r\right)^{\prime}$. If $\ell>n(1 / p+\tau+1 / 2)$, then there exists a positive constant $C$ such that for all $f \in$ $B \dot{H}_{p, r}^{0, \tau}\left(\mathbb{R}^{n}\right)$,

$$
\left\|T_{m} f\right\|_{B \dot{H}_{p * r}^{\beta, \tau}\left(\mathbb{R}^{n}\right)} \leq C\|f\|_{B \dot{H}_{, r, r}^{0, \tau}\left(\mathbb{R}^{n}\right)}
$$

Moreover, similar to Corollary 1.6(i), we can further improve the range of $\ell$ in Corollary 1.11(i) as follows.

Theorem 1.12. Let $\alpha \in \mathbb{R}, p \in(1, \infty), \beta \in \mathbb{R}$ with $\beta<\alpha$ and $p_{*} \in(1, \infty)$ such that $\beta-n / p_{*}=$ $\alpha-n / p$. Let $r, q \in(1, \infty)$ and $\tau \in\left[0, \min \left\{1 /(p \vee r)^{\prime}, 1 /\left(p_{*} \vee q\right)^{\prime}\right\}\right]$ such that $\tau(p \vee r)^{\prime} \leq \tau\left(p_{*} \vee q\right)^{\prime}$. Assume that $m$ satisfies (1.2) with $\ell \in \mathbb{N}$ and

$$
\ell> \begin{cases}n\left(\tau+\frac{1}{2}\right), & \text { if } \tau(p \vee r)^{\prime} \leq 2 \tau, \\ n\left(\frac{1}{r}+\frac{1}{2}\right), & \text { if } p, r \in(1,2), \quad \tau \neq 0 .\end{cases}
$$

Then there exists a positive constant $C$ such that for all $f \in F \dot{H}_{p, r}^{0, \tau}\left(\mathbb{R}^{n}\right)$,

$$
\left\|T_{m} f\right\|_{F \dot{H}_{p *, q}^{\beta, \tau}\left(\mathbb{R}^{n}\right)} \leq C\|f\|_{F \dot{H}_{,, r}^{0, \tau}\left(\mathbb{R}^{n}\right)} .
$$

The proof of Theorem 1.12 is given in Section 3.

Similar to Corollary 1.8, we have the following conclusion, which is an immediate consequence of Theorem 1.12 and the lifting property of the space $F \dot{H}_{p, q}^{s, \tau}\left(\mathbb{R}^{n}\right)$ that can be deduced directly from [15, Theorem 4.1]. 
Corollary 1.13. Let $\alpha, \gamma \in \mathbb{R}$ and $p, r, q \in(1, \infty)$. Let $\beta$ be a real number with $\beta<\alpha+\gamma$ and $p_{*} \in$ $(1, \infty)$ such that $\beta-n / p_{*}=\alpha+\gamma-n / p$. Assume that $\tau \in\left[0, \min \left\{1 /\left(p_{*} \vee q\right)^{\prime}, 1 /(p \vee r)^{\prime}\right\}\right]$ satisfies that $\tau(p \vee r)^{\prime} \leq \tau\left(p_{*} \vee q\right)^{\prime}$ and $m$ satisfies (1.2) with $\ell$ as in (1.27). Then there exists a positive constant $C$ such that for all $f \in F \dot{H}_{p, r}^{\gamma, \tau}\left(\mathbb{R}^{n}\right),\left\|T_{m} f\right\|_{F \dot{H}_{p * q}^{\beta, \tau}\left(\mathbb{R}^{n}\right)} \leq C\|f\|_{F \dot{H}_{p, r}^{\gamma, \tau}\left(\mathbb{R}^{n}\right)}$.

Corollary 1.13 implies that Theorem 1.12 has variant for any $s \in \mathbb{R}$ instead of $s=0$.

Remark 1.14. Recall that when $\tau=0$, the Triebel-Lizorkin-Hausdorff space $F \dot{H}_{p, q}^{s, \tau}\left(\mathbb{R}^{n}\right)$ is just the classical Triebel-Lizorkin space $\dot{F}_{p, q}^{s}\left(\mathbb{R}^{n}\right)$. Thus, when $\tau=0$, Theorem 1.12 coincides with Theorem 1.7. In this sense, Theorem 1.12 when $\tau=0$ also essentially improves [12, Theorem 5.2]; see Remark 1.9(iv).

The proofs of Theorems 1.5, 1.7, and 1.10 strongly depend on the Peetre-type maximal function characterizations of $\dot{B}_{p, q}^{s, \tau}\left(\mathbb{R}^{n}\right), \dot{F}_{p, q}^{s, \tau}\left(\mathbb{R}^{n}\right), B \dot{H}_{p, q}^{s, \tau}\left(\mathbb{R}^{n}\right)$, and $F \dot{H}_{p, q}^{s, \tau}\left(\mathbb{R}^{n}\right)$ obtained in [18]. Additionally, to prove Theorems 1.7 and 1.12, we need first establish the generalized (weighted) $g_{\curlywedge}^{*}$-function equivalent characterizations of $\dot{F}_{p, q}^{s, \tau}\left(\mathbb{R}^{n}\right)$ and $F \dot{H}_{p, q}^{s, \tau}\left(\mathbb{R}^{n}\right)$, respectively, in Theorems 2.7 and 2.9 below. We point out that Theorems 2.7 and 2.9 consist of two parts: sufficiency part and necessary part. The proofs of the sufficiency part are essentially deduced from the corresponding generalized Lusin-area function characterizations, obtained in [18], of these function spaces. The approach used in the proofs of the necessary part of Theorems 2.7 and 2.9 is totally different from that used in the proof of $\left[12\right.$, Lemma 3.2(3)] for $\dot{F}_{p, q}^{s}\left(\mathbb{R}^{n}\right)$, which induces an essential improvement of [12, Lemma 3.2(3)] such that we can replace the restriction $\lambda>n[\max (1 / p, 1 / r)]$ in $[12$, Lemma 3.2(3)] by $\lambda>n / r$. The proof of $[12$, Lemma 3.2(3)] strongly depends on the exact equivalent relations between the $L^{p}\left(\mathbb{R}^{n}\right)$ norms of the generalized Lusin-area functions with different apertures, which is not clear whether it is still true if $L^{p}\left(\mathbb{R}^{n}\right)$ norm is replaced by the Morrey norm. Instead of that, in the proofs of Theorems 2.7 and 2.9, we use the Lusin-area function characterization of these spaces and the homogeneity of the Euclidean space $\mathbb{R}^{n}$. This improvement further induces an improvement of Theorems 1.7 and 1.12 even when $\tau=0$, compared to [12, Theorem 5.2].

To prove Theorems 1.7 and 1.12, we need two technical lemmas from [12, Lemmas 4.1 and 4.2] (see also Lemmas 3.2 and 3.5 below). However, [12, Lemma 4.1(2)] therein is not accurate; see Remark 3.3 below. We give a corrected version in Lemma 3.2(ii) of this paper. We also remark that there exists a gap in the proof of [12, Theorem 5.2] for Triebel-Lizorkin spaces in the endpoint case when $p_{*}=\infty$; see Remark 1.9(iv). In this paper, we seal this gap via a subtle application of the equivalence between the Triebel-Lizorkin space $\dot{F}_{\infty, q}^{s}\left(\mathbb{R}^{n}\right)$ and the Triebel-Lizorkin-type space $\dot{F}_{p, q}^{s, 1 / p}\left(\mathbb{R}^{n}\right)$ obtained by Frazier and Jawerth [30, Corollary 5.7] (see also [14, Proposition 3.1]).

The paper is organized as follows. In Section 2, we present Theorems 2.7 and 2.9 and their proofs by first recalling some known characterizations, obtained in [18], of $\dot{B}_{p, q}^{s, \tau}\left(\mathbb{R}^{n}\right)$, $\dot{F}_{p, q}^{s, \tau}\left(\mathbb{R}^{n}\right), B \dot{H}_{p, q}^{s, \tau}\left(\mathbb{R}^{n}\right)$, and $F \dot{H}_{p, q}^{s, \tau}\left(\mathbb{R}^{n}\right)$ in terms of the Peetre-type maximal function and the Lusin-area function of local means. Section 3 is devoted to the proofs of Theorems 1.5, 1.7, 1.10, and 1.12. Finally in Section 4, as an application, we give a new proof of the Sobolev-type embedding theorems for $\dot{F}_{p, q}^{s, \tau}\left(\mathbb{R}^{n}\right)$ and $F \dot{H}_{p, q}^{s, \tau}\left(\mathbb{R}^{n}\right)$.

We point out that so far, for the Besov-type space $\dot{B}_{p, q}^{s, \tau}\left(\mathbb{R}^{n}\right)$ and the Besov-Hausdorff space $B \dot{H}_{p, q}^{s, \tau}\left(\mathbb{R}^{n}\right)$, it is unclear whether the corresponding results of Theorems 1.7 and 1.12 are true or not. The proofs of Theorems 1.7 and 1.12 strongly depend on the generalized (weighted) $g_{\lambda}^{*}$-function equivalent characterizations of $\dot{F}_{p, q}^{s, \tau}\left(\mathbb{R}^{n}\right)$ and $F \dot{H}_{p, q}^{s, \tau}\left(\mathbb{R}^{n}\right)$, which are not available for $\dot{B}_{p, q}^{s, \tau}\left(\mathbb{R}^{n}\right)$ and $B \dot{H}_{p, q}^{s, \tau}\left(\mathbb{R}^{n}\right)$. Moreover, it is also interesting to establish the inhomogeneous variants of these results. 
Finally, we make more conventions on the notation. Throughout the whole paper, the symbol $A \lesssim B$ means that $A \leq C B$, where $C$ is a positive constant independent of the main parameter. If $A \lesssim B$ and $B \lesssim A$, then we write $A \sim B$. If $E$ is a subset of $\mathbb{R}$, we denote by $\chi_{E}$ the characteristic function of $E$.

\section{Some Equivalent Characterizations of $\dot{F}_{p, q}^{s, \tau}\left(\mathbb{R}^{n}\right), \dot{B}_{p, q}^{s, \tau}\left(\mathbb{R}^{n}\right)$, $F \dot{H}_{p, q}^{s, \tau}\left(\mathbb{R}^{n}\right)$, and $B \dot{H}_{p, q}^{s, \tau}\left(\mathbb{R}^{n}\right)$}

In this section, we first recall some equivalent characterizations, established in [18], of $\dot{F}_{p, q}^{s, \tau}\left(\mathbb{R}^{n}\right), \dot{B}_{p, q}^{s, \tau}\left(\mathbb{R}^{n}\right), F \dot{H}_{p, q}^{s, \tau}\left(\mathbb{R}^{n}\right)$, and $B \dot{H}_{p, q}^{s, \tau}\left(\mathbb{R}^{n}\right)$, in terms of the Peetre-type maximal function and the Lusin-area function of local means. Using these characterizations, we further establish some new characterizations of these spaces in terms of the generalized (weighted) $g_{\Lambda^{*}}^{*}$ functions, which play a key role in the proofs of Theorems 1.7 and 1.12 in Section 3.

Let $\varepsilon \in(0, \infty), R \in \mathbb{Z}_{+} \cup\{-1\}$ and $\Phi \in \mathcal{S}\left(\mathbb{R}^{n}\right)$ satisfy that

$$
|\widehat{\Phi}(\xi)|>0 \quad \text { on }\left\{\xi \in \mathbb{R}^{n}: \frac{\varepsilon}{2}<|\xi|<2 \varepsilon\right\}, \quad D^{\alpha}(\widehat{\Phi})(0)=0 \quad \forall|\alpha| \leq R .
$$

In what follows, for any function $\varphi, t \in(0, \infty)$ and $x \in \mathbb{R}^{n}, \varphi_{t}(x):=t^{-n} \varphi(x / t)$. For all $\varphi \in$ $\mathcal{S}_{N}\left(\mathbb{R}^{n}\right), f \in \mathcal{S}_{N}^{\prime}\left(\mathbb{R}^{n}\right), t \in(0, \infty), \lambda \in(0, \infty)$, and $x \in \mathbb{R}^{n}$, let

$$
\left(\varphi_{t}^{*} f\right)_{\lambda}(x):=\sup _{y \in \mathbb{R}^{n}} \frac{\left|\left(\varphi_{t} * f\right)(x+y)\right|}{(1+|y| / t)^{\lambda}},
$$

which is called the Peetre-type maximal function of local means; see, for example, [18].

The following characterization of $\dot{F}_{p, q}^{s, \tau}\left(\mathbb{R}^{n}\right)$ was obtained in [18].

Theorem 2.1. Let $s \in \mathbb{R}, \tau \in[0, \infty), p \in(0, \infty), q \in(0, \infty], \lambda \in(n(1 / p \vee 1 / q), \infty)$ and $R \in$ $\mathbb{Z}_{+} \cup\{-1\}$ such that $s+n \tau<R+1$ and $\Phi$ be as in (2.1). Then the space $\dot{F}_{p, q}^{s, \tau}\left(\mathbb{R}^{n}\right)$ is characterized by

$$
\dot{F}_{p, q}^{s, \tau}\left(\mathbb{R}^{n}\right)=\left\{f \in \mathcal{S}_{R}^{\prime}\left(\mathbb{R}^{n}\right):\left\|f \mid \dot{F}_{p, q}^{s, \tau}\left(\mathbb{R}^{n}\right)\right\|_{i}<\infty\right\}, \quad i \in\{1,2,3\},
$$

where

$$
\begin{gathered}
\left\|f \mid \dot{F}_{p, q}^{s, \tau}\left(\mathbb{R}^{n}\right)\right\|_{1}:=\sup _{P \in \mathcal{Q}} \frac{1}{|P|^{\tau}}\left\{\int_{P}\left[\int_{0}^{\ell(P)} t^{-s q}\left|\left(\Phi_{t} * f\right)(x)\right|^{q} \frac{d t}{t}\right]^{p / q} d x\right\}^{1 / p}, \\
\left\|f \mid \dot{F}_{p, q}^{s, \tau}\left(\mathbb{R}^{n}\right)\right\|_{2}:=\sup _{P \in \mathcal{Q}} \frac{1}{|P|^{\tau}}\left\{\int_{P}\left[\int_{0}^{\ell(P)} t^{-s q}\left|\left(\Phi_{t}^{*} f\right)_{\lambda}(x)\right|^{q} \frac{d t}{t}\right]^{p / q} d x\right\}^{1 / p}, \\
\left\|f \mid \dot{F}_{p, q}^{s, \tau}\left(\mathbb{R}^{n}\right)\right\|_{3}:=\sup _{P \in \mathcal{Q}} \frac{1}{|P|^{\tau}}\left\{\int_{P}\left[\int_{0}^{\ell(P)} t^{-s q} \int_{|z|<t}\left|\left(\Phi_{t} * f\right)(x+z)\right|^{q} \frac{d z d t}{t^{n+1}}\right]^{p / q} d x\right\}^{1 / p},
\end{gathered}
$$

with the usual modification made when $q=\infty$. 
Remark 2.2. Recall that when $\tau \in[0,1 / p)$, the Triebel-Lizorkin-type spaces are just the TriebelLizorkin-Morrey spaces, that is, in the definition of Triebel-Lizorkin-type space, the sum $\sum_{j=j_{p}}^{\infty}$ can be replaced by $\sum_{j \in \mathbb{Z}}$; see [16, Theorem 1.1]. By an argument similar to that used in [18, Theorem 3.1], we can prove that Theorem 2.1 is also true with $\ell(P)$ replaced by $\infty$ in $\| f \mid$ $\dot{F}_{p, q}^{s, \tau}\left(\mathbb{R}^{n}\right)\left\|_{1},\right\| f \mid \dot{F}_{p, q}^{s, \tau}\left(\mathbb{R}^{n}\right) \|_{2}$ and $\left\|f \mid \dot{F}_{p, q}^{s, \tau}\left(\mathbb{R}^{n}\right)\right\|_{3}$ when $\tau \in[0,1 / p)$. We omit the details.

The following Theorems 2.3 through 2.5 were established in [18].

Theorem 2.3. Let $s \in \mathbb{R}, \tau \in[0, \infty), p, q \in(0, \infty], \lambda \in(n / p, \infty)$, and $R \in \mathbb{Z}_{+} \cup\{-1\}$ such that $s+n \tau<R+1$ and $\Phi$ be as in (2.1). Then the space $\dot{B}_{p, q}^{s, \tau}\left(\mathbb{R}^{n}\right)$ is characterized by

$$
\dot{B}_{p, q}^{s, \tau}\left(\mathbb{R}^{n}\right)=\left\{f \in \mathcal{S}_{R}^{\prime}\left(\mathbb{R}^{n}\right):\left\|f \mid \dot{B}_{p, q}^{s, \tau}\left(\mathbb{R}^{n}\right)\right\|_{i}<\infty\right\}, \quad i \in\{1,2\}
$$

where

$$
\begin{aligned}
& \left\|f \mid \dot{B}_{p, q}^{s, \tau}\left(\mathbb{R}^{n}\right)\right\|_{1}:=\sup _{P \in \mathcal{Q}} \frac{1}{|P|^{\tau}}\left\{\int_{0}^{\ell(P)} t^{-s q}\left[\int_{P}\left|\left(\Phi_{t} * f\right)(x)\right|^{p} d x\right]^{q / p} \frac{d t}{t}\right\}^{1 / q}, \\
& \left\|f \mid \dot{B}_{p, q}^{s, \tau}\left(\mathbb{R}^{n}\right)\right\|_{2}:=\sup _{P \in \mathcal{Q}} \frac{1}{|P|^{\tau}}\left\{\int_{0}^{\ell(P)} t^{-s q}\left[\int_{P}\left|\left(\Phi_{t}^{*} f\right)_{\lambda}(x)\right|^{p} d x\right]^{q / p} \frac{d t}{t}\right\}^{1 / q},
\end{aligned}
$$

with the usual modifications made when $q=\infty$ or $p=\infty$.

Theorem 2.4. Let $s \in \mathbb{R}, p, q \in(1, \infty), \tau \in\left[0,1 /(p \vee q)^{\prime}\right], \lambda \in(n[\max \{1 / p, 1 / q\}+\tau], \infty)$ and $R \in \mathbb{Z}_{+} \cup\{-1\}$ such that $s+n \tau<R+1$ and $\Phi$ be as in (2.1). Then the space $F \dot{H}_{p, q}^{s, \tau}\left(\mathbb{R}^{n}\right)$ is characterized by

$$
F \dot{H}_{p, q}^{s, \tau}\left(\mathbb{R}^{n}\right)=\left\{f \in \mathcal{S}_{R}^{\prime}\left(\mathbb{R}^{n}\right):\left\|f \mid F \dot{H}_{p, q}^{s, \tau}\left(\mathbb{R}^{n}\right)\right\|_{i}<\infty\right\}, \quad i \in\{1,2,3\},
$$

where

$$
\begin{gathered}
\left\|f \mid F \dot{H}_{p, q}^{s, \tau}\left(\mathbb{R}^{n}\right)\right\|_{1}:=\inf _{\omega}\left\|\left\{\int_{0}^{\infty} t^{-s q}\left|\Phi_{t} * f\right|^{q}[\omega(\cdot, t)]^{-q} \frac{d t}{t}\right\}^{1 / q}\right\|_{L^{p}\left(\mathbb{R}^{n}\right)}, \\
\left\|f \mid F \dot{H}_{p, q}^{s, \tau}\left(\mathbb{R}^{n}\right)\right\|_{2}:=\inf _{\omega}\left\|\left\{\int_{0}^{\infty} t^{-s q}\left[\left(\Phi_{t}^{*} f\right)_{\lambda}\right]^{q}[\omega(\cdot, t)]^{-q} \frac{d t}{t}\right\}^{1 / q}\right\|_{L^{p}\left(\mathbb{R}^{n}\right)}, \\
\left\|f \mid F \dot{H}_{p, q}^{s, \tau}\left(\mathbb{R}^{n}\right)\right\|_{3}:=\inf _{\omega}\left\|\left\{\int_{0}^{\infty} t^{-s q} \int_{|z|<t}\left|\Phi_{t} * f(\cdot+z)\right|^{q}[\omega(\cdot+z, t)]^{-q} \frac{d z d t}{t^{n+1}}\right\}^{1 / q}\right\|_{L^{p}\left(\mathbb{R}^{n}\right)},
\end{gathered}
$$

where the infimums are taken over all nonnegative Borel measurable functions $\omega$ on $\mathbb{R}_{+}^{n+1}$ satisfying (1.13). 
Theorem 2.5. Let $s \in \mathbb{R}, p \in(1, \infty), q \in[1, \infty), \tau \in\left[0,1 /(p \vee q)^{\prime}\right], \lambda \in(n(1 / p+\tau), \infty)$ and $R \in \mathbb{Z}_{+} \cup\{-1\}$ such that $s+n \tau<R+1$ and $\Phi$ be as in (2.1). Then the space $B \dot{H}_{p, q}^{s, \tau}\left(\mathbb{R}^{n}\right)$ is characterized by

$$
B \dot{H}_{p, q}^{s, \tau}\left(\mathbb{R}^{n}\right)=\left\{f \in \mathcal{S}_{R}^{\prime}\left(\mathbb{R}^{n}\right):\left\|f \mid B \dot{H}_{p, q}^{s, \tau}\left(\mathbb{R}^{n}\right)\right\|_{i}<\infty\right\}, \quad i \in\{1,2\},
$$

where

$$
\begin{aligned}
& \left\|f \mid B \dot{H}_{p, q}^{s, \tau}\left(\mathbb{R}^{n}\right)\right\|_{1}:=\inf _{\omega}\left\{\int_{0}^{\infty} t^{-s q}\left\|\Phi_{t} * f[\omega(\cdot, t)]^{-1}\right\|_{L^{p}\left(\mathbb{R}^{n}\right)}^{q} \frac{d t}{t}\right\}^{1 / q}, \\
& \left\|f \mid B \dot{H}_{p, q}^{s, \tau}\left(\mathbb{R}^{n}\right)\right\|_{2}:=\inf _{\omega}\left\{\int_{0}^{\infty} t^{-s q}\left\|\left(\Phi_{t}^{*} f\right)_{\lambda}[\omega(\cdot, t)]^{-1}\right\|_{L^{p}\left(\mathbb{R}^{n}\right)}^{q} \frac{d t}{t}\right\}^{1 / q},
\end{aligned}
$$

where the infimums are taken over all nonnegative Borel measurable functions $\omega$ on $\mathbb{R}_{+}^{n+1}$ satisfying (1.13).

Remark 2.6. (i) The space $F \dot{H}_{p, q}^{s, \tau}\left(\mathbb{R}^{n}\right)$ is a quasi-Banach space; see [13, 14, 17]. Indeed, by [17, Remarks 7.1 and 7.3], we know that for any $f_{1}, f_{2} \in F \dot{H}_{p, q}^{s, \tau}\left(\mathbb{R}^{n}\right)$,

$$
\left\|f_{1}+f_{2}\right\|_{F \dot{H}_{p, q}^{s, \tau}\left(\mathbb{R}^{n}\right)} \leq 2^{1 /(p \vee q)^{\prime}}\left[\left\|f_{1}\right\|_{F \dot{H}_{p, q}^{s, \tau}\left(\mathbb{R}^{n}\right)}+\left\|f_{2}\right\|_{F \dot{H}_{p, q}^{s, \tau}\left(\mathbb{R}^{n}\right)}\right]
$$

(ii) By the Aoki-Rolewicz theorem $([35,36])$, there exists $v \in(0,1]$ such that

$$
\left\|\sum_{j \in \mathbb{Z}} f_{j}\right\|_{F \dot{H}_{p, q}^{s, \tau}\left(\mathbb{R}^{n}\right)}^{v} \lesssim \sum_{j \in \mathbb{Z}}\left\|f_{j}\right\|_{F \dot{H}_{p, q}^{s, \tau}\left(\mathbb{R}^{n}\right)}^{v}
$$

for all $\left\{f_{j}\right\}_{j \in \mathbb{Z}} \subset F \dot{H}_{p, q}^{s, \tau}\left(\mathbb{R}^{n}\right)$. Indeed, $v:=(p \vee q)^{\prime} /\left(1+(p \vee q)^{\prime}\right)$ does the job.

(iii) The conclusions in (i) and (ii) are also true for the space $B \dot{H}_{p, q}^{s, \tau}\left(\mathbb{R}^{n}\right)$.

Next we establish a new characterization of the spaces $\dot{F}_{p, q}^{s, \tau}\left(\mathbb{R}^{n}\right)$ and $F \dot{H}_{p, q}^{s, \tau}\left(\mathbb{R}^{n}\right)$. Let $q \in(0, \infty], \lambda \in(0, \infty)$, and $\omega$ be a nonnegative Borel measurable function. In what follows, for $R \in \mathbb{Z}_{+} \cup\{-1\}, f \in \mathcal{S}_{R}^{\prime}\left(\mathbb{R}^{n}\right)$ and $\varphi$ in (2.1), set

$$
u(x, t):=\left(f * \varphi_{t}\right)(x), \quad u_{\lambda}^{*}(x, t):=\sup _{y \in \mathbb{R}^{n}}\left\{|u(y, t)|\left(1+\frac{|x-y|}{t}\right)^{-\lambda}\right\}
$$


for all $x \in \mathbb{R}^{n}$ and $t \in(0, \infty)$. For all $b \in(0, \infty), s \in \mathbb{R}$, and $x \in \mathbb{R}^{n}$, recall that the generalized weighted Lusin-area function $S_{b, q}^{s}(\omega, u)(x)$ and the generalized weighted $g_{\lambda}^{*}$-function $G_{\lambda, q}^{s}(\omega, u)(x)$ are defined, respectively, by

$$
\begin{gathered}
S_{b, q}^{s}(\omega, u)(x):=\left\{\int_{0}^{\infty} t^{-s q} \int_{|y-x|<b t}|u(y, t)|^{q}[\omega(y, t)]^{-q}(b t)^{-n} d y \frac{d t}{t}\right\}^{1 / q}, \\
G_{\lambda, q}^{s}(\omega, u)(x):=\left\{\int_{0}^{\infty} t^{-s q} \int_{\mathbb{R}^{n}}|u(y, t)|^{q}\left(1+\frac{|x-y|}{t}\right)^{-\lambda q}[\omega(y, t)]^{-q} d y \frac{d t}{t^{n+1}}\right\}^{1 / q} .
\end{gathered}
$$

If $\omega(x, t) \equiv 1$, then $S_{b, q}^{s}(\omega, u)$ and $G_{\lambda, q}^{s}(\omega, u)$ are called, respectively, the generalized Lusin-area function, denoted by $S_{b, q}^{s}(u)$, and the generalized $g_{\lambda}^{*}$-function, denoted by $G_{\lambda, q}^{s}(u)$.

In what follows, for $\tau \in[0, \infty)$ and $p \in(0, \infty)$, let $L_{\tau}^{p}\left(\mathbb{R}^{n}\right)$ be the set of all functions $f \in$ $L_{\text {loc }}^{p}\left(\mathbb{R}^{n}\right)$ such that

$$
\|f\|_{L_{\tau}^{p}\left(\mathbb{R}^{n}\right)}:=\sup _{p \in \mathcal{Q}} \frac{1}{|P|^{\tau}}\left[\int_{P}|f(x)|^{p} d x\right]^{1 / p}<\infty
$$

Theorem 2.7. Let $s \in \mathbb{R}, p \in(0, \infty), \tau \in[0,1 / p), q \in(0, \infty], \lambda \in(n / q, \infty)$ and $R \in \mathbb{Z}_{+} \cup\{-1\}$ such that $s+n \tau<R+1$. Then $f \in \dot{F}_{p, q}^{s, \tau}\left(\mathbb{R}^{n}\right)$ if and only if $f \in \mathcal{S}_{R}^{\prime}\left(\mathbb{R}^{n}\right)$ and $G_{\lambda, q}^{s}(u) \in L_{\tau}^{p}\left(\mathbb{R}^{n}\right)$, where $u$ is as in (2.13). Moreover, there exists a positive constant $C$ such that for all $f \in \dot{F}_{p, q}^{s, \tau}\left(\mathbb{R}^{n}\right)$,

$$
C^{-1}\|f\|_{\dot{F}_{p, q}^{s, \tau}\left(\mathbb{R}^{n}\right)} \leq\left\|G_{\lambda, q}^{s}(u)\right\|_{L_{\tau}^{p}\left(\mathbb{R}^{n}\right)} \leq C\|f\|_{\dot{F}_{p, q}^{s, \tau}\left(\mathbb{R}^{n}\right)}
$$

Proof. Assume $f \in \mathcal{S}_{R}^{\prime}\left(\mathbb{R}^{n}\right)$ and $G_{\lambda, q}^{s}(u) \in L_{\tau}^{p}\left(\mathbb{R}^{n}\right)$. Notice that for any $\lambda \in(0, \infty)$ and $x \in \mathbb{R}^{n}$,

$$
\begin{aligned}
S_{1, q}^{s}(u)(x) & \leq 2^{\lambda}\left\{\int_{0}^{\infty} \int_{|y-x|<t}\left[t^{-s}|u(y, t)|\right]^{q}\left(1+\frac{|x-y|}{t}\right)^{-\lambda q} t^{-n} d y \frac{d t}{t}\right\}^{1 / q} \\
& =2^{\lambda} G_{\lambda, q}^{s}(u)(x) .
\end{aligned}
$$

Then, from Remark 2.2, we deduce that $f \in \dot{F}_{p, q}^{s, \tau}\left(\mathbb{R}^{n}\right)$ and

$$
\|f\|_{\dot{F}_{p, q}^{s, \tau}\left(\mathbb{R}^{n}\right)} \sim\left\|S_{1, q}^{s}(u)\right\|_{L_{\tau}^{p}\left(\mathbb{R}^{n}\right)} \lesssim\left\|G_{\lambda, q}^{s}(u)\right\|_{L_{\tau}^{p}\left(\mathbb{R}^{n}\right)^{\prime}}
$$

which completes the proof of the sufficiency of the theorem. 
Conversely, suppose that $f \in \dot{F}_{p, q}^{s, \tau}\left(\mathbb{R}^{n}\right)$. Then by Theorem 2.1, $f \in \mathcal{S}_{R}^{\prime}\left(\mathbb{R}^{n}\right)$. Moreover, similar to the proof of $[18$, Theorem 3.1], for any $k \in \mathbb{N}$, we see that

$$
\begin{gathered}
\sup _{P \in \mathcal{Q}} \frac{1}{|P|^{\tau}}\left\{\int_{P}\left[\int_{0}^{\infty} \int_{|y-x|<t}\left(t^{-s}\left|\varphi_{2^{-k} t} * f(y)\right|\right)^{q} t^{-n} d y \frac{d t}{t}\right]^{p / q} d x\right\}^{1 / p} \\
\leq \operatorname{Csup}_{P \in \mathcal{Q}} \frac{1}{|P|^{\tau}}\left\{\int_{P}\left[\sum_{j=-\infty}^{\infty} 2^{j s q}\left|\varphi_{k+j} * f(x)\right|^{q}\right]^{p / q} d x\right\}^{1 / p},
\end{gathered}
$$

where $C$ is a positive constant independent of $k$ and $f$. Then by changing variables, we conclude that

$$
\begin{aligned}
\sup _{P \in \mathcal{Q}} & \frac{1}{|P|^{\tau}}\left\{\int_{P}\left[\int_{0}^{\infty} \int_{|y-x|<t}\left(t^{-s}\left|\varphi_{2-k} * f(y)\right|\right)^{q} t^{-n} d y \frac{d t}{t}\right]^{p / q} d x\right\}^{1 / p} \\
& \lesssim 2^{-k s} \sup _{P \in \mathcal{Q}} \frac{1}{|P|^{\tau}}\left\{\int_{P}\left[\sum_{j=-\infty}^{\infty} 2^{j s q}\left|\varphi_{j} * f(x)\right|^{q}\right]^{p / q} d x\right\}^{1 / p} \\
& \lesssim 2^{-k s}\|f\|_{\dot{F}_{p, q}^{s, \tau}\left(\mathbb{R}^{n}\right)^{\prime}}
\end{aligned}
$$

where the last inequality follows from the equivalence between Triebel-Lizorkin spaces and Triebel-Lizorkin-Morrey spaces when $\tau \in[0,1 / p)$; see [16] and also Remark 2.2. By changing variables, we know that for all $x \in \mathbb{R}^{n}$,

$$
\begin{aligned}
G_{\lambda, q}^{s}(u)(x)= & \left\{\int _ { 0 } ^ { \infty } \left[\int_{|y-x|<t}\left(t^{-s}\left|\varphi_{t} * f(y)\right|\right)^{q}\left(1+\frac{|x-y|}{t}\right)^{-\lambda q} t^{-n} d y\right.\right. \\
& \left.\left.+\sum_{k=1}^{\infty} \int_{2^{k-1} t \leq|y-x|<2^{k} t} \cdots d y\right] \frac{d t}{t}\right\}^{1 / q} \\
\lesssim & \left\{\sum_{k=0}^{\infty} 2^{-k \lambda q} \int_{0}^{\infty} \int_{|y-x|<2^{k} t}\left(t^{-s}\left|\varphi_{t} * f(y)\right|\right)^{q} t^{-n} d y \frac{d t}{t}\right\}^{1 / q} \\
\lesssim & \left\{\sum_{k=0}^{\infty} 2^{-k(\lambda q-s q-n)} \int_{0}^{\infty} \int_{|y-x|<t}\left(t^{-s}\left|\varphi_{2^{-k} t} * f(y)\right|\right)^{q} t^{-n} d y \frac{d t}{t}\right\}^{1 / q} .
\end{aligned}
$$

Thus, when $p \leq q$, from the well-known inequality that for all $d \in(0,1]$ and $\left\{\alpha_{j}\right\}_{j} \subset \mathbb{C}$,

$$
\left(\sum_{j}\left|\alpha_{j}\right|\right)^{d} \leq \sum_{j}\left|\alpha_{j}\right|^{d}
$$


it follows that

$$
\begin{aligned}
\left\|G_{\lambda, q}^{s}(u)\right\|_{L_{\tau}^{p}\left(\mathbb{R}^{n}\right)} & \lesssim \sup _{P \in \mathcal{Q}} \frac{1}{|P|^{\tau}}\left\{\int_{P}\left[\sum_{k=0}^{\infty} 2^{-k(\lambda q-s q-n)} \int_{0}^{\infty} \int_{|y-x|<t}\left(t^{-s}\left|\varphi_{2^{-k} t} * f(y)\right|\right)^{q} t^{-n} d y \frac{d t}{t}\right]^{p / q} d x\right\}^{1 / p} \\
& \lesssim \sup _{P \in \mathcal{Q}} \frac{1}{|P|^{\tau}}\left\{\int_{P} \sum_{k=0}^{\infty} 2^{-k(\lambda-s-n / q) p}\left[\int_{0}^{\infty} \int_{|y-x|<t}\left(t^{-s}\left|\varphi_{2^{-k} t} * f(y)\right|\right)^{q} t^{-n} d y \frac{d t}{t}\right]^{p / q} d x\right\}^{1 / p} \\
& \lesssim\left\{\sum_{k=0}^{\infty} 2^{-k(\lambda-s-n / q) p}\right. \\
& \left.\times \sup _{P \in \mathcal{Q}} \frac{1}{|P|^{\tau p}} \int_{P}\left[\int_{0}^{\infty} \int_{|y-x|<t}\left(t^{-s}\left|\varphi_{2^{-k} t} * f(y)\right|\right)^{q} t^{-n} d y \frac{d t}{t}\right]^{p / q} d x\right\}^{1 / p} \\
& \lesssim\left\{\sum_{k=0}^{\infty} 2^{-k(\lambda-n / q) p}\|f\|_{F_{p, q}^{s, \tau}\left(\mathbb{R}^{n}\right)}^{p}\right\}^{1 / p} \lesssim\|f\|_{\dot{F}_{p, q}^{s, \tau}\left(\mathbb{R}^{n}\right)^{\prime}}
\end{aligned}
$$

where the last inequality follows from (2.20) and $\lambda>n / q$. Similarly, when $p>q$, by Minkowski's inequality and (2.20), we see that

$$
\begin{aligned}
& \left\|G_{\lambda, q}^{s}(u)\right\|_{L_{\tau}^{p}\left(\mathbb{R}^{n}\right)} \\
& \qquad \sup _{P \in \mathcal{Q}} \frac{1}{|P|^{\tau}}\left(\sum_{k=0}^{\infty} 2^{-k(\lambda q-s q-n)}\right. \\
& \left.\quad \times\left\{\int_{P}\left[\int_{0}^{\infty} \int_{|y-x|<t}\left(t^{-s}\left|\varphi_{2^{-k} t} * f(y)\right|\right)^{q} t^{-n} d y \frac{d t}{t}\right]^{p / q} d x\right\}^{q / p}\right)^{1 / q} \\
& \lesssim\left(\sum_{k=0}^{\infty} 2^{-k(\lambda q-s q-n)} \sup _{P \in \mathcal{Q}} \frac{1}{|P|^{\tau q}}\right. \\
& \left.\quad \times\left\{\int_{P}\left[\int_{0}^{\infty} \int_{|y-x|<t}\left(t^{-s}\left|\varphi_{2^{-k} t} * f(y)\right|\right)^{q} t^{-n} d y \frac{d t}{t}\right]^{p / q} d x\right\}^{q / p}\right)^{1 / q} \\
& \lesssim\left\{\sum_{k=0}^{\infty} 2^{-k(\lambda q-n)}\|f\|_{\dot{F}_{p, q}^{s, \tau}\left(\mathbb{R}^{n}\right)}^{q}\right\}^{1 / q} \lesssim\|f\|_{\dot{F}_{p, q}^{s, \tau}\left(\mathbb{R}^{n}\right)^{.}}
\end{aligned}
$$


These estimates, together with Remark 2.2, imply the necessity of the theorem and hence complete the proof of Theorem 2.7.

Remark 2.8. We point that, by an argument similar to the proof of Theorem 2.7, one can characterize $\dot{F}_{p, q}^{s, \tau}\left(\mathbb{R}^{n}\right)$ via a discrete version of the generalized weighted $g_{l}^{*}$-function. More precisely, for all $s \in \mathbb{R}, p \in(0, \infty), \tau \in[0,1 / p), q \in(0, \infty], \lambda \in(n / q, \infty)$, and $R \in \mathbb{Z}_{+} \cup\{-1\}$ such that $s+n \tau<R+1$, then $f \in \dot{F}_{p, q}^{s, \tau}\left(\mathbb{R}^{n}\right)$ if and only if $f \in \mathcal{S}_{R}^{\prime}\left(\mathbb{R}^{n}\right)$ and $\left[\sum_{i \in \mathbb{Z}} \int_{\mathbb{R}^{n}} 2^{i(s q+n)}\left|f * \varphi_{i}(z)\right|^{q}\left(1+2^{i}|z-\cdot|\right)^{-\lambda q} d z\right]^{1 / q} \in L_{\tau}^{p}\left(\mathbb{R}^{n}\right)$. Moreover,

$$
\|f\|_{\dot{F}_{p, q}^{s, \tau}\left(\mathbb{R}^{n}\right)} \sim\left\|\left[\sum_{i \in \mathbb{Z}} \int_{\mathbb{R}^{n}} 2^{i(s q+n)}\left|f * \varphi_{i}(z)\right|^{q}\left(1+2^{i}|z-\cdot|\right)^{-\lambda q} d z\right]^{1 / q}\right\|_{L_{\tau}^{p}\left(\mathbb{R}^{n}\right)}
$$

We omit the details.

We also obtain the following analogy of Theorem 2.7 for the space $F \dot{H}_{p, q}^{s, \tau}\left(\mathbb{R}^{n}\right)$.

Theorem 2.9. Let $s \in \mathbb{R}, p \in(1, \infty), q \in(1, \infty], \tau \in\left[0,1 /(p \vee q)^{\prime}\right], \lambda \in(n / q, \infty)$ and $R \in$ $\mathbb{Z}_{+} \cup\{-1\}$ such that $s+n \tau<R+1$. Then $f \in F \dot{H}_{p, q}^{s, \tau}\left(\mathbb{R}^{n}\right)$ if and only if $f \in \mathcal{S}_{R}^{\prime}\left(\mathbb{R}^{n}\right)$ and $G_{\lambda, q}^{s}(\omega, u) \in$ $L^{p}\left(\mathbb{R}^{n}\right)$, where $u$ is as in (2.13). Moreover, there exists a positive constant $C$ such that for all $f \in$ $F \dot{H}_{p, q}^{s, \tau}\left(\mathbb{R}^{n}\right)$,

$$
C^{-1}\|f\|_{F \dot{H}_{p, q}^{s, \tau}\left(\mathbb{R}^{n}\right)} \leq \inf _{\omega}\left\|G_{\lambda, q}^{s}(\omega, u)\right\|_{L^{p}\left(\mathbb{R}^{n}\right)} \leq C\|f\|_{F \dot{H}_{p, q}^{s, \tau}\left(\mathbb{R}^{n}\right)^{\prime}}
$$

where the infimum is taken over all nonnegative Borel measurable functions $\omega$ on $\mathbb{R}_{+}^{n+1}$ satisfying (1.13).

Proof. Assume $f \in \mathcal{S}_{R}^{\prime}\left(\mathbb{R}^{n}\right)$ and $G_{\lambda, q}^{s}(\omega, u) \in L^{p}\left(\mathbb{R}^{n}\right)$. For any $\lambda \in(0, \infty)$ and $x \in \mathbb{R}^{n}$, similar to the proof of Theorem 2.7, we know that

$$
S_{1, q}^{s}(\omega, u)(x) \leq 2^{\lambda q} G_{\lambda, q}^{s}(\omega, u)(x) .
$$

Then by Theorem 2.4, we see that

$$
\|f\|_{F H_{p, q}^{s, \tau}\left(\mathbb{R}^{n}\right)} \sim \inf _{\omega}\left\|S_{1, q}^{s}(\omega, u)\right\|_{L^{p}\left(\mathbb{R}^{n}\right)} \lesssim \inf _{\omega}\left\|G_{\lambda, q}^{s}(\omega, u)\right\|_{L^{p}\left(\mathbb{R}^{n}\right)^{\prime}}
$$

which completes the proof of the sufficiency of the theorem. 
Conversely, suppose that $f \in F \dot{H}_{p, q}^{s, \tau}\left(\mathbb{R}^{n}\right)$. Then by Theorem 1.12, $f \in \mathcal{S}_{R}^{\prime}\left(\mathbb{R}^{n}\right)$. By an argument similar to the proof of [18, Theorem 3.3], we see that for any $k \in \mathbb{N}$,

$$
\begin{aligned}
\inf _{\omega} \| & \left\{\int_{0}^{\infty} \int_{|z-\cdot|<t} t^{-s q}\left|\left(\left(\varphi_{k}\right)_{t} * f\right)(z)\right|^{q}\left[\omega\left(z, 2^{-k} t\right)\right]^{-q} d z \frac{d t}{t^{n+1}}\right\}^{1 / q} \|_{L^{p}\left(\mathbb{R}^{n}\right)} \\
& \lesssim \inf _{\omega}\left\|\left\{\sum_{j \in \mathbb{Z}} 2^{j s q}\left|\varphi_{k+j} * f\right|^{q}\left[\omega\left(\cdot, 2^{-k-j}\right)\right]^{-q}\right\}^{1 / q}\right\| \|_{L^{p}\left(\mathbb{R}^{n}\right)} \\
& \sim 2^{-k s} \inf _{\omega}\left\|\left\{\sum_{j \in \mathbb{Z}} 2^{j s q}\left|\varphi_{j} * f\right|^{q}\left[\omega\left(\cdot, 2^{-j}\right)\right]^{-q}\right\}^{1 / q}\right\|_{L^{p}\left(\mathbb{R}^{n}\right)} \\
& \sim 2^{-k s}\|f\|_{F \dot{H}_{p, q}^{s, \tau}\left(\mathbb{R}^{n}\right)^{.}}
\end{aligned}
$$

Let $\mathbb{R}_{+}:=(0, \infty)$. For all measurable functions $F$ on $\mathbb{R}^{n} \times \mathbb{R}_{+} \times \mathbb{R}^{n}$, let

$$
\|F\|_{\mathcal{F}}:=\inf _{\omega}\left\|\left\{\int_{0}^{\infty} t^{-s q} \int_{\mathbb{R}^{n}}|F(y, t, \cdot)|[\omega(y, t)]^{-q} d y \frac{d t}{t^{n+1}}\right\}^{1 / q}\right\|_{L^{p}\left(\mathbb{R}^{n}\right)},
$$

where the infimum is taken over the same set as in (1.13). We claim that $\|\cdot\|_{\mathscr{F}}$ is a quasinorm with respect to $F$, precisely, for any measurable functions $F_{1}, F_{2}$ on $\mathbb{R}^{n} \times \mathbb{R}_{+} \times \mathbb{R}^{n}$,

$$
\left\|F_{1}+F_{2}\right\|_{\mathcal{F}} \leq 2^{1 /(p \vee q)^{\prime}}\left(\left\|F_{1}\right\|_{\mathcal{F}}+\left\|F_{2}\right\|_{\mathcal{F}}\right) .
$$

To see this, without loss of generality, we may assume that $\left\|F_{1}\right\|_{\mathcal{F}}+\left\|F_{2}\right\|_{\mathcal{F}}<\infty$. Then, for any $\varepsilon \in(0, \infty)$, choose nonnegative Borel measurable functions $\omega_{1}, \omega_{2}$ on $\mathbb{R}_{+}^{n+1}$ satisfying (1.13) such that

$$
\left\|\left\{\int_{0}^{\infty} t^{-s q} \int_{\mathbb{R}^{n}}\left|F_{i}(y, t, \cdot)\right|\left[\omega_{i}(y, t)\right]^{-q} d y \frac{d t}{t^{n+1}}\right\}^{1 / q}\right\|_{L^{p}\left(\mathbb{R}^{n}\right)} \leq(1+\varepsilon)\left\|F_{i}\right\|_{\mathcal{F}},
$$


Journal of Function Spaces and Applications

for $i \in\{1,2\}$. Notice that $\omega:=2^{-1 /(p \vee q)^{\prime}} \max \left\{\omega_{1}, \omega_{2}\right\}$ still satisfies (1.13). Then by (2.22) and Minkowski's inequality, we see that

$$
\begin{aligned}
\left\|F_{1}+F_{2}\right\|_{\mathcal{F}} \leq & \left\|\left\{\int_{0}^{\infty} t^{-s q} \int_{\mathbb{R}^{n}}\left|F_{1}(y, t, \cdot)+F_{2}(y, t, \cdot)\right|[\omega(y, t)]^{-q} d y \frac{d t}{t^{n+1}}\right\}^{1 / q}\right\|_{L^{p}\left(\mathbb{R}^{n}\right)} \\
\leq & 2^{1 /(p v q)^{\prime}}\left[\left\|\left\{\int_{0}^{\infty} t^{-s q} \int_{\mathbb{R}^{n}}\left|F_{1}(y, t, \cdot)\right|\left[\omega_{1}(y, t)\right]^{-q} d y \frac{d t}{t^{n+1}}\right\}^{1 / q}\right\|_{L^{p}\left(\mathbb{R}^{n}\right)}\right. \\
& \left.+\left\|\left\{\int_{0}^{\infty} t^{-s q} \int_{\mathbb{R}^{n}}\left|F_{2}(y, t, \cdot)\right|\left[\omega_{2}(y, t)\right]^{-q} d y \frac{d t}{t^{n+1}}\right\}^{1 / q}\right\|_{L^{p}\left(\mathbb{R}^{n}\right)}\right] \\
\leq & 2^{1 /(p \vee q)^{\prime}}(1+\varepsilon)\left(\left\|F_{1}\right\|_{\mathcal{F}}+\left\|F_{2}\right\|_{\mathcal{F}}\right) .
\end{aligned}
$$

Letting $\varepsilon \rightarrow 0$ then concludes the above claim.

Thus, by the Aoki-Rolewica theorem $[35,36]$, we know that

$$
\left\|\sum_{j \in \mathbb{Z}} F_{j}\right\|_{\mp}^{v} \lesssim \sum_{j \in \mathbb{Z}}\left\|F_{j}\right\|_{\mathcal{F}^{\prime}}^{v}
$$

for all measurable functions $\left\{F_{j}\right\}_{j \in \mathbb{Z}}$ on $\mathbb{R}^{n} \times \mathbb{R}_{+} \times \mathbb{R}^{n}$, where $v=(p \vee q)^{\prime} /\left(1+(p \vee q)^{\prime}\right)$.

Choosing $\lambda>n / q$, by (2.34), (2.29), and an estimate similar to (2.21), we conclude that

$$
\begin{aligned}
\inf _{\omega}\left\|G_{\lambda, q}^{s}(\omega, u)\right\|_{L^{p}\left(\mathbb{R}^{n}\right)}^{v} & \\
& =\inf _{\omega}\left\|\left\{\int_{0}^{\infty} t^{-s q} \int_{\mathbb{R}^{n}}\left|\varphi_{t} * f(y)\right|^{q}\left(1+\frac{|\cdot-y|}{t}\right)^{-\lambda q}[\omega(y, t)]^{-q} d y \frac{d t}{t^{n+1}}\right\}^{1 / q}\right\|_{L^{p}\left(\mathbb{R}^{n}\right)}^{v} \\
& \lesssim \inf _{\omega}\left\|\left\{\int_{0}^{\infty} t^{-s q} \sum_{k=0}^{\infty} \int_{B\left(\cdot, 2^{k} t\right)} 2^{-\lambda k q}\left|\varphi_{t} * f(y)\right|^{q}[\omega(y, t)]^{-q} d y \frac{d t}{t^{n+1}}\right\}^{1 / q}\right\|_{L^{p}\left(\mathbb{R}^{n}\right)}^{v} \\
& \lesssim \sum_{k=0}^{\infty} 2^{-\lambda k v} \inf _{\omega}\left\|\left\{\int_{0}^{\infty} t^{-s q} \int_{\mathbb{R}^{n}}\left|\varphi_{t} * f(y)\right|^{q} X_{B\left(\cdot 2^{k} t\right)}(y)[\omega(y, t)]^{-q} d y \frac{d t}{t^{n+1}}\right\}^{1 / q}\right\|_{L^{p}\left(\mathbb{R}^{n}\right)}^{v} \\
& \lesssim \sum_{k=0}^{\infty} 2^{-k(\lambda-s-n / q) v}
\end{aligned}
$$




$$
\begin{aligned}
& \times \inf _{\omega}\left\|\left[\int_{0}^{\infty} \int_{|y-\cdot|<t} t^{-s q}\left|\left(\varphi_{k}\right)_{t} * f(y)\right|^{q}\left[\omega\left(y, 2^{-k} t\right)\right]^{-q} d y \frac{d t}{t^{n+1}}\right]^{1 / q}\right\|_{L^{p}\left(\mathbb{R}^{n}\right)}^{v} \\
& \lesssim \sum_{k=0}^{\infty} 2^{-k(\lambda-n / q) v}\|f\|_{F \dot{H}_{p, q}^{s, \tau}\left(\mathbb{R}^{n}\right)}^{v} \sim\|f\|_{F \dot{H}_{p, q}^{s, \tau}\left(\mathbb{R}^{n}\right)^{\prime}}^{v}
\end{aligned}
$$

which implies the necessity of the theorem and hence completes the proof of Theorem 2.9.

\section{Proofs of Theorems 1.5, 1.7, 1.10, and 1.12}

In this section, we give the proofs for Theorems 1.5, 1.7, 1.10, and 1.12.

In what follows, $K$ always denotes the distribution whose Fourier transform is the function $m$ in (1.2). Then we have the following observation.

Lemma 3.1. Let $m$ be as in (1.2) and $K$ its inverse Fourier transform. Then $K \in \mathcal{S}_{\infty}^{\prime}\left(\mathbb{R}^{n}\right)$.

Proof. Let $\varphi \in \mathcal{S}_{\infty}\left(\mathbb{R}^{n}\right)$. Then $\partial^{\gamma} \hat{\varphi}(0)=0$ for all $\gamma \in \mathbb{Z}_{+}^{n}$ and hence for any $L \in \mathbb{N}$,

$$
\begin{aligned}
\langle K, \varphi\rangle & =\int_{\mathbb{R}^{n}} m(\xi) \widehat{\varphi}(\xi) d \xi \\
& =\int_{|\xi| \geq 1} m(\xi) \widehat{\varphi}(\xi) d \xi+\int_{|\xi|<1} m(\xi)\left[\widehat{\varphi}(\xi)-\sum_{|\gamma| \leq L} \frac{\partial^{\gamma} \widehat{\varphi}(0)}{\gamma !} \xi\right] d \xi=: \mathrm{I}_{1}+\mathrm{I}_{2} .
\end{aligned}
$$

For $\mathrm{I}_{1}$, by Hölder's inequality and (1.2), we see that

$$
\begin{aligned}
\left|\mathrm{I}_{1}\right| & \lesssim \sum_{k=0}^{\infty} \int_{2^{k} \leq|\xi|<2^{k+1}}|m(\xi)||\widehat{\varphi}(\xi)| d \xi \\
& \lesssim \sum_{k=0}^{\infty} \frac{1}{\left(1+2^{k}\right)^{M}} \int_{2^{k} \leq|\xi|<2^{k+1}}|m(\xi)| d \xi \\
& \lesssim \sum_{k=0}^{\infty} \frac{2^{n k / 2}}{\left(1+2^{k}\right)^{M}}\left[\int_{2^{k} \leq|\xi|<2^{k+1}}|m(\xi)|^{2} d \xi\right]^{1 / 2} \\
& \lesssim \sum_{k=0}^{\infty} \frac{2^{k(n-\alpha)}}{\left(1+2^{k}\right)^{M}} \lesssim 1,
\end{aligned}
$$

where $M \in[0, \infty)$ is chosen large enough such that $M>n-\alpha$. 
For $\mathrm{I}_{2}$, by the mean value theorem, there exists $\theta \in[0,1]$ such that

$$
\begin{aligned}
\left|\mathrm{I}_{2}\right| & \lesssim \sum_{k=-\infty}^{-1} \int_{2^{k} \leq|\xi|<2^{k+1}}|m(\xi)| \sup _{|\gamma|=L+1}\left|\partial^{\gamma} \widehat{\varphi}(\theta \xi)\right||\xi|^{|\gamma|} d \xi \\
& \lesssim \sum_{k=-\infty}^{-1} 2^{k(L+1)} \int_{2^{k} \leq|\xi|<2^{k+1}}|m(\xi)| d \xi \\
& \lesssim \sum_{k=-\infty}^{-1} 2^{k(L+1+n-\alpha)} \lesssim 1
\end{aligned}
$$

where $L$ is chosen large enough such that $L>\alpha-n-1$. This finishes the proof of Lemma 3.1 . and 1.12 .

The following estimates play an important role in the proofs of Theorems 1.5, 1.7, 1.10,

Lemma 3.2. Let $\psi$, $\zeta$ be Schwartz functions on $\mathbb{R}^{n}$ such that $\widehat{\psi}, \widehat{\zeta}$ are supported in the annulus $\left\{\xi \in \mathbb{R}^{n}: 1 / 2 \leq|\xi| \leq 2\right\}$. Assume that $m$ satisfies (1.2).

(i) If $\lambda \in(0, \infty)$ and $\ell>\lambda+n / 2$, then there exists a positive constant $C$ such that for all $t \in(0, \infty)$,

$$
\int_{\mathbb{R}^{n}}\left(1+\frac{|z|}{t}\right)^{\curlywedge}\left|\left(K * \psi_{t}\right)(z)\right| d z \leq C t^{\alpha}
$$

(ii) Let $k, N$ be any two positive integers. If $\ell \geq \lambda>0$, then there exists a positive constant $C$ such that for all $s, t \in(0, \infty)$,

$$
\int_{\mathbb{R}^{n}}\left(1+\frac{|z|}{s}\right)^{2 \lambda}\left|\left(K * \zeta_{s} * \psi_{t}\right)(z)\right|^{2} d z \leq C(\min \{t, s\})^{-n+2 \alpha} \begin{cases}\left(1+\frac{t}{s}\right)^{-2 N}, & \text { if } s \leq t, \\ \left(\frac{t}{s}\right)^{2 k}, & \text { if } t<s .\end{cases}
$$

Remark 3.3. We remark that Lemma 3.2(i) is just [12, Lemma 4.1(1)]. It was also claimed in $[12$, Lemma $4.1(2)]$ that the inequality in Lemma 3.2(ii) is valid with $(\min \{t, s\})^{-n+2 \alpha}$ replaced by $s^{-n+2 \alpha}$. However, the proof of [12, Lemma 4.1(2)] is problematic. Indeed, the last inequality in [12, page 849] seems to be true only when $s \leq t$. We give a correct version in Lemma 3.2(ii). 
Proof of Lemma 3.2(ii). Since $\lambda \leq \ell$, by the Plancherel theorem, we see that

$$
\begin{aligned}
\int_{\mathbb{R}^{n}} & \left(1+\frac{|z|}{s}\right)^{2 \lambda}\left|\left(K * \zeta_{s} * \psi_{t}\right)(z)\right|^{2} d z \\
& \lesssim \sum_{|\sigma| \leq \ell} s^{-2|\sigma|} \int_{\mathbb{R}^{n}}\left|\partial_{\xi}^{\sigma}\left(m(\xi) \widehat{\zeta}(\xi) \widehat{\psi_{t}}(\xi)\right)\right|^{2} d \xi \\
& \lesssim \sum_{|\sigma| \leq \ell} s^{-2|\sigma|} \sum_{\sigma_{1}+\sigma_{2}+\sigma_{3}=\sigma} s^{2\left|\sigma_{2}\right|} t^{2\left|\sigma_{3}\right|} \int_{\mathbb{R}^{n}}\left|\left(\partial_{\xi}^{\sigma_{1}} m\right)(\xi)\left(\partial_{\xi}^{\sigma_{2}} \widehat{\zeta}\right)(s \xi)\left(\partial_{\xi}^{\sigma_{3}} \widehat{\psi}\right)(t \xi)\right|^{2} d \xi
\end{aligned}
$$

When $s \leq t$, by the support of $\widehat{\zeta}$ and (1.2), we see that

$$
\begin{aligned}
\int_{\mathbb{R}^{n}} & \left(1+\frac{|z|}{s}\right)^{2 \lambda}\left|\left(K * \zeta_{s} * \psi_{t}\right)(z)\right|^{2} d z \\
& \lesssim \sum_{|\sigma| \leq \ell} s^{-2|\sigma|} \sum_{\sigma_{1}+\sigma_{2}+\sigma_{3}=\sigma} s^{2\left|\sigma_{2}\right|} t^{2\left|\sigma_{3}\right|} \int_{1 /(2 s) \leq|\xi| \leq 2 / s}\left|\left(\partial_{\xi}^{\sigma_{1}} m\right)(\xi)\left(\partial_{\xi}^{\sigma_{3}} \widehat{\psi}\right)(t \xi)\right|^{2} d \xi \\
& \lesssim \sum_{|\sigma| \leq \ell} \sum_{\sigma_{1}+\sigma_{2}+\sigma_{3}=\sigma}\left(\frac{t}{s}\right)^{2\left|\sigma_{3}\right|} \frac{1}{(1+t / s)^{2 N+2 \ell}} s^{-2\left|\sigma_{1}\right|} \int_{1 /(2 s) \leq|\xi| \leq 2 / s}\left|\left(\partial_{\xi}^{\sigma_{1}} m\right)(\xi)\right|^{2} d \xi \\
& \lesssim s^{-n+2 \alpha} \frac{1}{(1+t / s)^{2 N}} .
\end{aligned}
$$

When $t \leq s$, by the support of $\widehat{\psi}$ and (1.2), we conclude that

$$
\begin{aligned}
\int_{\mathbb{R}^{n}} & \left(1+\frac{|z|}{s}\right)^{2 \lambda}\left|\left(K * \zeta_{s} * \psi_{t}\right)(z)\right|^{2} d z \\
& \lesssim \sum_{|\sigma| \leq \ell} \sum_{\sigma_{1}+\sigma_{2}+\sigma_{3}=\sigma} s^{-2|\sigma|} s^{2\left|\sigma_{2}\right|} t^{2\left|\sigma_{3}\right|} \int_{1 /(2 t) \leq|\xi| \leq 2 / t}\left|\left(\partial_{\xi}^{\sigma_{1}} m\right)(\xi)\left(\partial_{\xi}^{\sigma_{2}} \widehat{\zeta}\right)(s \xi)\right|^{2} d \xi \\
& \lesssim \sum_{|\sigma| \leq \ell} \sum_{\sigma_{1}+\sigma_{2}+\sigma_{3}=\sigma}\left(\frac{t}{s}\right)^{2\left|\sigma_{1}\right|+2\left|\sigma_{3}\right|} \frac{1}{(1+(s / t))^{2 k}} t^{-2\left|\sigma_{1}\right|} \int_{1 /(2 t) \leq|\xi| \leq 2 / t}\left|\left(\partial_{\xi}^{\sigma_{1}} m\right)(\xi)\right|^{2} d \xi \\
& \lesssim t^{-n+2 \alpha}\left(\frac{t}{s}\right)^{2 k}
\end{aligned}
$$

which completes the proof of Lemma 3.5(ii).

Recall that $\mathcal{S}_{\infty}\left(\mathbb{R}^{n}\right)$ is dense in the spaces $F \dot{H}_{p, q}^{s, \tau}\left(\mathbb{R}^{n}\right)$ and $B \dot{H}_{p, q}^{s, \tau}\left(\mathbb{R}^{n}\right)$ (see $[13$, Lemma 5.3] and [14, Lemma 6.3]). Then the definition of Fourier multiplier $T_{m}$ can be extended to the whole spaces $F \dot{H}_{p, q}^{s, \tau}\left(\mathbb{R}^{n}\right)$ and $B \dot{H}_{p, q}^{s, \tau}\left(\mathbb{R}^{n}\right)$ via a dense argument. Next we show that, 
via a suitable way, $T_{m}$ can also be defined on the whole spaces $\dot{F}_{p, q}^{s, \tau}\left(\mathbb{R}^{n}\right)$ and $\dot{B}_{p, q}^{s, \tau}\left(\mathbb{R}^{n}\right)$. To this end, let $\varphi \in \mathcal{A}$. Then by [37, Lemma (6.9)], there exists $\psi \in \mathcal{A}$ such that

$$
\sum_{i \in \mathbb{Z}} \overline{\hat{\varphi}\left(2^{-i} \xi\right)} \widehat{\psi}\left(2^{-i} \xi\right)=1, \quad \forall \xi \in \mathbb{R}^{n} \backslash\{0\}
$$

For any $f \in \dot{F}_{p, q}^{s, \tau}\left(\mathbb{R}^{n}\right)$ or $\dot{B}_{p, q}^{s, \tau}\left(\mathbb{R}^{n}\right)$, we define $T_{m} f$ by setting, for all $\phi \in \mathcal{S}_{\infty}\left(\mathbb{R}^{n}\right)$,

$$
\left\langle T_{m} f, \phi\right\rangle:=\sum_{i \in \mathbb{Z}} f * \varphi_{i} * \psi_{i} * \phi * K(0) .
$$

In this sense, we say $T_{m} f \in \mathcal{S}_{\infty}^{\prime}\left(\mathbb{R}^{n}\right)$. The following result shows that $T_{m} f$ in (3.10) is well defined.

Lemma 3.4. Let $\ell \in(n / 2, \infty), s \in \mathbb{R}, \tau \in[0, \infty)$ and $q \in(0, \infty], f \in \dot{F}_{p, q}^{s, \tau}\left(\mathbb{R}^{n}\right)$ with $p \in(0, \infty)$ or $f \in \dot{B}_{p, q}^{s, \tau}\left(\mathbb{R}^{n}\right)$ with $p \in(0, \infty]$. Then $T_{m} f$ in $(3.10)$ is independent of the choices of the pair $(\varphi, \psi)$ of functions in A satisfying (3.9). Moreover, $T_{m} f \in \mathcal{S}_{\infty}^{\prime}\left(\mathbb{R}^{n}\right)$.

Proof. Assume first that $f \in \dot{F}_{p, \infty}^{s, \tau}\left(\mathbb{R}^{n}\right)$. Let $\tilde{\varphi}$ and $\tilde{\psi}$ be another pair of functions in $\mathbb{A}$ satisfying (3.9). Since $\phi \in \mathcal{S}_{\infty}\left(\mathbb{R}^{n}\right)$, by the Calderón reproducing formula (see [13, Lemma 2.1]), we know that

$$
\phi=\sum_{j \in \mathbb{Z}} \tilde{\varphi}_{j} * \widetilde{\psi} * \phi
$$

in $\mathcal{S}_{\infty}\left(\mathbb{R}^{n}\right)$. Thus,

$$
\begin{aligned}
\sum_{i \in \mathbb{Z}} f * \varphi_{i} * \psi_{i} * \phi * K(0) & =\sum_{i \in \mathbb{Z}} f * \varphi_{i} * \psi_{i} *\left(\sum_{j \in \mathbb{Z}} \tilde{\varphi}_{j} * \widetilde{\varphi}_{j} * \phi\right) * K(0) \\
& =\sum_{i \in \mathbb{Z}} \sum_{j=i-1}^{i+1} f *\left(\varphi_{i} * \tilde{\varphi}_{j}\right) *\left(\psi_{i} * \widetilde{\varphi}_{j}\right) * \phi * K(0),
\end{aligned}
$$

where the last equality follows from the fact that $\varphi_{i} * \tilde{\varphi}_{j}=0$ if $|i-j| \geq 2$. all $x \in \mathbb{R}^{n}$,

Let $\zeta:=\varphi * \tilde{\varphi}$ and $\eta:=\psi * \widetilde{\psi}$. Then $\zeta, \eta \in \mathcal{A}$. If $\tau \in[0,1 / p)$ and $q=\infty$, we see that for

$$
\begin{aligned}
& \sum_{i \in \mathbb{Z}}\left|f *\left(\varphi_{i} * \tilde{\varphi}_{i}\right) *\left(\psi_{i} * \widetilde{\varphi}_{i}\right) * \phi * K(0)\right| \\
& =\sum_{i \in \mathbb{Z}}\left|\int_{\mathbb{R}^{n}} f * \zeta_{i}(-z) \eta_{i} * \phi * K(z) d z\right|
\end{aligned}
$$




$$
\begin{aligned}
& \lesssim\left[\sup _{i \in \mathbb{Z}} \sup _{z \in \mathbb{R}^{n}} 2^{i s}\left|f * \zeta_{i}(-z)\right|\left(1+2^{i}|z+x|\right)^{-\lambda}\right] \\
& \times \sum_{i \in \mathbb{Z}} \int_{\mathbb{R}^{n}} 2^{-i s}\left(1+2^{i}|z+x|\right)^{\lambda}\left|K * \eta_{i} * \phi(z)\right| d z=: \mathrm{I}_{1}(x) \mathrm{I}_{2}(x),
\end{aligned}
$$

where $\lambda$ is an arbitrary positive number.

For $\mathrm{I}_{1}$, by Remark 2.8, we know that $\left\|\mathrm{I}_{1}\right\|_{L_{\tau}^{p}\left(\mathbb{R}^{n}\right)} \lesssim\|f\|_{F_{p, \infty}^{s, \pi}\left(\mathbb{R}^{n}\right)}<\infty$, which implies that there exists $x \in B(0,1)$ such that $\left|\mathrm{I}_{1}(x)\right|<\infty$.

For $\mathrm{I}_{2}$, choosing $\lambda \in(0, \infty)$ and $\mu \in(n / 2, \infty)$ such that $\lambda+\mu<\ell$, by Hölder's inequality and Lemma 3.2(ii), we see that for all $x \in B(0,1)$,

$$
\begin{aligned}
\int_{\mathbb{R}^{n}} & \left(1+2^{i}|z+x|\right)^{\lambda}\left|K * \eta_{i} * \phi(z)\right| d z \\
& \lesssim\left(1+2^{i}\right)^{\lambda} \int_{\mathbb{R}^{n}}\left(1+2^{i}|z|\right)^{\lambda}\left|K * \eta_{i} * \phi(z)\right| d z \\
& \lesssim\left(1+2^{i}\right)^{\lambda} 2^{-i n / 2}\left[\int_{\mathbb{R}^{n}}\left(1+2^{i}|z|\right)^{2(\lambda+\mu)}\left|K * \eta_{i} * \phi(z)\right|^{2} d z\right]^{1 / 2} \\
& \lesssim 2^{-i n / 2}\left(\min \left\{1,2^{-i}\right\}\right)^{-n / 2+\alpha}\left(\min \left\{1,2^{i}\right\}\right)^{k}\left(1+2^{i}\right)^{\lambda-N} .
\end{aligned}
$$

Thus, by choosing $k$ and $N$ large enough such that $k>n / 2+|s|$ and $N>\lambda+|\alpha|+|s|$, we know that

$$
\mathrm{I}_{2}(x) \lesssim \sum_{i=0}^{\infty} 2^{i(\lambda-\alpha-s-N)}+\sum_{i=-\infty}^{-1} 2^{i(k-n / 2-s)} \lesssim 1
$$

Therefore,

$$
\sum_{i \in \mathbb{Z}}\left|f *\left(\varphi_{i} * \tilde{\varphi}_{i}\right) *\left(\psi_{i} * \tilde{\varphi}_{i}\right) * \phi * K(0)\right|<\infty
$$

By an argument similar to the above, we see that

$$
\sum_{i \in \mathbb{Z}} \sum_{j=i-1}^{i+1}\left|f *\left(\varphi_{i} * \tilde{\varphi}_{j}\right) *\left(\psi_{i} * \widetilde{\varphi}_{j}\right) * \phi * K(0)\right|<\infty
$$

which, together with the Calderon reproducing formula, further induces that

$$
\begin{array}{rl}
\sum_{i \in \mathbb{Z}} f & * \varphi_{i} * \psi_{i} * \varphi * K(0) \\
& =\sum_{j \in \mathbb{Z}} \sum_{i=j-1}^{j+1} f *\left(\varphi_{i} * \tilde{\varphi}_{j}\right) *\left(\varphi_{i} * \widetilde{\varphi}_{j}\right) * \phi * K(0)
\end{array}
$$


Journal of Function Spaces and Applications

$$
\begin{aligned}
& =\sum_{j \in \mathbb{Z}} f * \tilde{\varphi}_{j} * \tilde{\varphi}_{j} *\left(\sum_{i \in \mathbb{Z}} \varphi_{i} * \psi_{i} * \phi\right) * K(0) \\
& =\sum_{j \in \mathbb{Z}} f * \tilde{\varphi}_{j} * \tilde{\varphi}_{j} * \varphi * K(0) .
\end{aligned}
$$

Thus, $T_{m} f$ in (3.10) is independent of the choices of the pair $(\varphi, \psi)$. Moreover, the previous argument also implies that $T_{m} f \in S_{\infty}^{\prime}\left(\mathbb{R}^{n}\right)$.

If $\tau \in[0,1 / p)$ and $q \in(0, \infty)$, from the embedding $\dot{F}_{p, q}^{s, \tau}\left(\mathbb{R}^{n}\right) \subset \dot{F}_{p, \infty}^{s, \tau}\left(\mathbb{R}^{n}\right)$, we deduce that $T_{m} f$ is also well defined in $\dot{F}_{p, q}^{s, \tau}\left(\mathbb{R}^{n}\right)$ and $T_{m} f \in \mathcal{S}_{\infty}^{\prime}\left(\mathbb{R}^{n}\right)$.

If $\tau \in(1 / p, \infty)$ and $q \in(0, \infty)$, by Remark 1.2 and [29, Corollary 1$]$, we know that

$$
\dot{F}_{p, q}^{s, \tau}\left(\mathbb{R}^{n}\right)=\dot{F}_{\infty, \infty}^{s+n(\tau-1 / p)}\left(\mathbb{R}^{n}\right)=\dot{B}_{\infty, \infty}^{s+n(\tau-1 / p)}\left(\mathbb{R}^{n}\right)
$$

Then, by Theorem 2.3, we know that

$$
\begin{aligned}
& \sum_{i \in \mathbb{Z}}\left|\int_{\mathbb{R}^{n}} f * \zeta_{i}(-z) \eta_{i} * \phi * K(z) d z\right| \\
& \quad \leq\left[\sup _{i \in \mathbb{Z}} \sup _{z \in \mathbb{R}^{n}} 2^{i[s+n(\tau-1 / p)]}\left|f * \zeta_{i}(z)\right|\right] \sum_{i \in \mathbb{Z}} 2^{-i[s+n(\tau-1 / p)]} \int_{\mathbb{R}^{n}}\left|\eta_{i} * \phi * K(z)\right| d z \\
& \quad \sim\|f\|_{\dot{F}_{\infty, \infty}^{s+n(\tau-1 / p)}\left(\mathbb{R}^{n}\right)} \sum_{i \in \mathbb{Z}} 2^{-i[s+n(\tau-1 / p)]} \int_{\mathbb{R}^{n}}\left|\eta_{i} * \phi * K(z)\right| d z .
\end{aligned}
$$

Choosing $\lambda>n / 2$ such that $\ell>\lambda$, by Hölder's inequality and Lemma 3.2(ii), we conclude that

$$
\begin{aligned}
\sum_{i \in \mathbb{Z}} 2^{-i[s+n(\tau-1 / p)]} \int_{\mathbb{R}^{n}}\left|\eta_{i} * \phi * K(z)\right| d z & \\
& \lesssim \sum_{i \in \mathbb{Z}} 2^{-i[s+n(\tau-1 / p)]}\left[\int_{\mathbb{R}^{n}}\left(1+2^{i}|z|\right)^{-2 \lambda} d z\right]^{1 / 2}\left[\int_{\mathbb{R}^{n}}\left(1+2^{i}|z|\right)^{2 \lambda}\left|\eta_{i} * \phi * K(z)\right|^{2} d z\right]^{1 / 2} \\
& \lesssim \sum_{i \in \mathbb{Z}} 2^{-i[s+n(\tau-1 / p+1 / 2)]}\left(\min \left\{1,2^{-i}\right\}\right)^{-n / 2+\alpha}\left(\min \left\{1,2^{i}\right\}\right)^{k}\left(1+2^{i}\right)^{-N} \\
& \lesssim \sum_{i=0}^{\infty} 2^{-i[s+n \tau-n / p+\alpha+N]}+\sum_{i=-\infty}^{-1} 2^{-i(s+n \tau-n / p+n / 2-k)} \lesssim 1,
\end{aligned}
$$


where $k$ and $N$ are chosen large enough such that $k>|s|+n(\tau-n / p+n / 2)$ and $N>|s|+|\alpha|+$ $n / p$. By an argument similar to the above, we see that

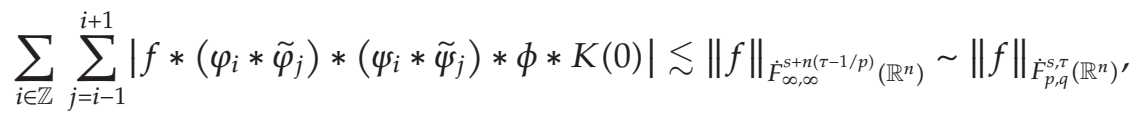

which, together with the Calderón reproducing formula, further induces that

$$
\sum_{i \in \mathbb{Z}} f * \varphi_{\mathrm{i}} * \psi_{i} * \phi * K(0)=\sum_{j \in \mathbb{Z}} f * \tilde{\varphi}_{j} * \tilde{\varphi}_{j} * \phi * K(0) .
$$

Thus, in the case that $\tau \in(1 / p, \infty), T_{m} f$ in (3.10) is also independent of the choices of the pair $(\varphi, \psi)$. Moreover, $T_{m} f \in \mathcal{S}_{\infty}^{\prime}\left(\mathbb{R}^{n}\right)$.

Finally, if $\tau=1 / p$ and $q \in(0, \infty)$, since $\dot{F}_{p, q}^{s, 1 / p}\left(\mathbb{R}^{n}\right)=\dot{F}_{\infty, q}^{s}\left(\mathbb{R}^{n}\right) \subset \dot{F}_{\infty, \infty}^{s}\left(\mathbb{R}^{n}\right)$ (see [30, Corollary 5.7]), from the previous argument, we deduce that $T_{m}$ is also well defined in $\dot{F}_{p, q}^{s, 1 / p}\left(\mathbb{R}^{n}\right)$. Therefore, we obtain the desired conclusion for the space $\dot{F}_{p, q}^{s, \tau}\left(\mathbb{R}^{n}\right)$ for all admissible indices.

Assume now that $f \in \dot{B}_{p, q}^{s, \tau}\left(\mathbb{R}^{n}\right)$. If $p \in(0, \infty)$, by the obtained conclusion for $\dot{F}_{p, q}^{s, \tau}\left(\mathbb{R}^{n}\right)$, the embedding $\dot{B}_{p, q}^{s, \tau}\left(\mathbb{R}^{n}\right) \subset \dot{F}_{p, q}^{s, \tau}\left(\mathbb{R}^{n}\right)$ when $q \leq p$ (see [14, Proposition 3.1(vii)]) and

$$
\dot{B}_{p, q}^{s, \tau}\left(\mathbb{R}^{n}\right) \subset \dot{B}_{p, p}^{s-\epsilon, \tau+\epsilon / n}\left(\mathbb{R}^{n}\right) \subset \dot{F}_{p, q}^{s-\epsilon, \tau+\epsilon / n}\left(\mathbb{R}^{n}\right)
$$

for some $\epsilon$ when $q>p$ (see (iii) and (vii) of [14, Proposition 3.1]), we know that $T_{m}$ is well defined in $\dot{B}_{p, q}^{s, \tau}\left(\mathbb{R}^{n}\right)$. This, together with the embedding $\dot{B}_{\infty, q}^{s, \tau}\left(\mathbb{R}^{n}\right) \subset \dot{B}_{p_{0}, q}^{s, \tau+1 / p_{0}}\left(\mathbb{R}^{n}\right)$ for some $p_{0} \in$ $(0, \infty)$ (see [14, Proposition 3.1(ii)]), further induces the corresponding result for $\dot{B}_{\infty, q}^{s, \tau}\left(\mathbb{R}^{n}\right)$, and hence completes the proof of Lemma 3.4.

Now we have the following technical lemma.

Lemma 3.5. Let $\alpha \in \mathbb{R}, \lambda \in(0, \infty), r \in[2, \infty], \ell \in \mathbb{N}, \varphi, \psi \in \mathcal{A}$, and $u$, $u_{\lambda}^{*}$ be as in (2.13). Assume that $m$ satisfies (1.2) and $f \in S_{\infty}^{\prime}\left(\mathbb{R}^{n}\right)$ such that $T_{m} f \in S_{\infty}^{\prime}\left(\mathbb{R}^{n}\right)$.

(i) If $\ell>\lambda+n / 2$ and $\Phi=\varphi * \psi$, then for all $x, y \in \mathbb{R}^{n}$ and $t \in(0, \infty)$,

$$
\left|\left(T_{m} f * \Phi_{t}\right)(y)\right| \leq C t^{\alpha}\left(1+\frac{|x-y|}{t}\right)^{\lambda} u_{\lambda}^{*}(x, t) .
$$

(ii) If $\ell>\lambda+n(1 / 2-1 / r)$, then for all $x, y \in \mathbb{R}^{n}$ and $t \in(0, \infty)$ satisfying that $|x-y|<t$,

$$
\left|\left(T_{m} f * \psi_{t}\right)(y)\right| \leq C t^{\alpha} G_{\lambda, r}^{0}(u)(x) .
$$

Proof. (i) is just [12, Lemma 4.2(1)]. The proof of (ii) is similar to the proofs of (2) and (3) of [12, Lemma 4.2], but with [12, Lemma 4.1(2)] replaced by Lemma 3.2(ii). This finishes the proof of Lemma 3.5. 
We remark that by Lemma 3.4, $T_{m} f \in S_{\infty}^{\prime}\left(\mathbb{R}^{n}\right)$ when $f \in \dot{F}_{p, q}^{s, \tau}\left(\mathbb{R}^{n}\right)$ or $\dot{B}_{p, q}^{s, \tau}\left(\mathbb{R}^{n}\right)$ with all indices as in Lemma 3.4. Thus, Lemma 3.5 is also true for all $f \in \dot{F}_{p, q}^{s, \tau}\left(\mathbb{R}^{n}\right)$ or $f \in \dot{B}_{p, q}^{s, \tau}\left(\mathbb{R}^{n}\right)$ with all indices as in Lemma 3.4.

Now we are ready to prove Theorems 1.5, 1.7, 1.10, and 1.12.

Proof of Theorem 1.5. Let $\Phi$ be as in Lemma 3.5.

(i) By the assumption that $\ell>n[\max (1 / p, 1 / r)+1 / 2]$, there exists $\lambda>n[\max (1 / p, 1 / r)]$ such that $\ell>\lambda+n / 2$. Then by Lemma 3.5(i), we see that for all $x \in \mathbb{R}^{n}$ and $t \in(0, \infty)$,

$$
t^{-(\alpha+\gamma)}\left(\Phi_{t}^{*}\left(T_{m} f\right)\right)_{\lambda}(x) \lesssim t^{-\gamma} u_{\lambda}^{*}(x, t)
$$

which yields the desired result in view of Theorem 2.1.

(ii) By the assumption that $\ell>n(1 / p+1 / 2)$, there exists $\lambda>n / p$ such that $\ell>\lambda+n / 2$. Then by Lemma 3.5(i), we also see that for all $x \in \mathbb{R}^{n}$ and $t \in(0, \infty)$, (3.27) holds, which yields the desired result in view of Theorem 2.1 and hence completes the proof of Theorem 1.5.

Now we give the proof of Theorem 1.7.

Proof of Theorem 1.7. To prove the theorem, by the monotone embedding property on the parameter $q$ of the spaces $\dot{F}_{p_{*}, q}^{\beta, \tau}\left(\mathbb{R}^{n}\right)$ (see [14, Proposition 3.1(i)]), namely, $\dot{F}_{p_{*}, q_{1}}^{\beta, \tau}\left(\mathbb{R}^{n}\right) \subset \dot{F}_{p_{*}, q_{2}}^{\beta, \tau}\left(\mathbb{R}^{n}\right)$ if $q_{1} \leq q_{2}$, it suffices to consider the case $q \in(0, \infty)$. We show the desired result in two cases for $\tau$.

Case $1(\tau \in[0,1 / p))$. Assume first that $f \in \dot{F}_{p, r}^{0, \tau}\left(\mathbb{R}^{n}\right)$ with $r \in[2, \infty]$. By assumption that $\ell>$ $n / 2$, we know that there exists $\lambda>n / r$ such that $\ell>\lambda+n / 2-n / r$. Then from Lemma 3.5(ii), we deduce that for all $x, y \in \mathbb{R}^{n}$ and $t \in(0, \infty)$ satisfying that $|x-y|<t$,

$$
|U(y, t)| \lesssim t^{\alpha} G_{\lambda, r}^{0}(u)(x)
$$

where and in what follows, $U(x, t):=\left(T_{m} f * \psi_{t}\right)(x)$ for all $x \in \mathbb{R}^{n}$ and $t \in(0, \infty)$.

If $\|f\|_{F_{p, r}^{0, r}\left(\mathbb{R}^{n}\right)}=0$, by Theorem 2.7, we know that $\left\|G_{\lambda, r}^{0}(u)\right\|_{L_{\tau}^{p}\left(\mathbb{R}^{n}\right)}=0$, and hence $G_{\lambda, r}^{0}(u)(x)=0$ for almost every $x \in \mathbb{R}^{n}$, which, together with (3.28), implies that $U(y, t)=0$ for all $y \in \mathbb{R}^{n}$. We then conclude that $\left\|T_{m} f\right\|_{\dot{F}_{p *, q}^{\beta, \tau}\left(\mathbb{R}^{n}\right)}=0$.

If $\|f\|_{\hat{F}_{p, r}^{0, \tau}\left(\mathbb{R}^{n}\right)}>0$, we know that $\left\|G_{\lambda, r}^{0}(u)\right\|_{L_{\tau}^{p}\left(\mathbb{R}^{n}\right)}>0$. Let $P$ be a dyadic cube and $t \in$ $(0, \ell(P))$. Then, there exist $3^{n}$ dyadic cubes $\left\{P_{i}\right\}_{i=1}^{3^{n}}$, with $\ell\left(P_{i}\right)=\ell(P)$, such that

$$
\{y: \operatorname{dist}(y, P)<t\} \subset \bigcup_{i=1}^{3^{n}} P_{i} .
$$


Then, raising (3.28) to the power $p$ and integrating over the ball $B(y, t)$, we see that

$$
\begin{aligned}
\left\{\int_{B(y, t)}|U(y, t)|^{p} d x\right\}^{1 / p} & \lesssim t^{\alpha}\left\{\int_{\bigcup_{i=1}^{3^{n}} P_{i}}\left|G_{\lambda, r}^{0}(u)(x)\right|^{p} d x\right\}^{1 / p} \\
& \lesssim t^{\alpha} \sum_{i=1}^{3^{n}}\left\{\int_{P_{i}}\left|G_{\lambda, r}^{0}(u)(x)\right|^{p} d x\right\}^{1 / p},
\end{aligned}
$$

which further implies that

$$
|U(y, t)| \lesssim t^{\alpha-n / p} \sum_{i=1}^{3^{n}}\left\{\int_{P_{i}}\left|G_{\lambda, r}^{0}(u)(x)\right|^{p} d x\right\}^{1 / p} .
$$

For any fixed $x \in P$ and $A:=A(x) \in(0, \infty)$ which is determined later, by (3.28), (3.31), $\alpha>\beta$ and $\alpha-\beta-n / p=-n / p_{*}$, we see that

$$
\begin{aligned}
\int_{0}^{\ell(P)} & t^{-\beta q} \int_{|y-x|<t}|U(y, t)|^{q} t^{-n} d y \frac{d t}{t} \\
& =\int_{0}^{A} \int_{|y-x|<t} X(0, \ell(P)) \\
& \lesssim\left[G_{\lambda, r}(u)(x) t^{-\beta q}|U(y, t)|^{q} t^{-n} d y \frac{d t}{t}+\int_{A}^{\infty} \int_{|y-x|<t} \cdots d y \frac{d t}{t}\right. \\
& \lesssim\left[G_{\lambda, r}^{0}(u)(x)\right]^{q} A^{(\alpha-\beta) q}+\| \sum_{i=1}^{3^{n}}\left\{\left.\int_{P_{i}}\left|G_{\lambda, r}^{0}(u) \|_{L_{\tau}^{p}\left(\mathbb{R}^{n}\right)}^{q}\right| P\right|^{\tau q} A^{(\alpha-\beta-n / p) q} .\right.
\end{aligned}
$$

Take $A$ such that

$$
A^{-n / p}=\frac{G_{\lambda, r}^{0}(u)(x)}{\left(|P|^{\tau}\left\|G_{\lambda, r}^{0}(u)\right\|_{L_{\tau}^{p}\left(\mathbb{R}^{n}\right)}\right)} .
$$

Then we see that

$$
\begin{aligned}
& \left\{\int_{0}^{\ell(P)} t^{-\beta q} \int_{|y-x|<t}|U(y, t)|^{q} t^{-n} d y \frac{d t}{t}\right\}^{1 / q} \\
& \quad \lesssim|P|^{\tau\left(1-p / p_{*}\right)}\left\|G_{\lambda, r}^{0}(u)\right\|_{L_{\tau}^{p}\left(\mathbb{R}^{n}\right)}^{1-p / p_{*}}\left[G_{\lambda, r}^{0}(u)(x)\right]^{p / p_{*}} .
\end{aligned}
$$


Then, by Theorem 2.7 and $\lambda>n / r$, we conclude that

$$
\begin{aligned}
\left\|T_{m} f\right\|_{\dot{F}_{p *, q}^{\beta, \tau}\left(\mathbb{R}^{n}\right)} & =\sup _{P \in \mathcal{Q}} \frac{1}{|P|^{\tau}}\left\{\int_{P}\left(\int_{0}^{\ell(P)} \int_{|y-x| \leq t}\left[t^{-\beta}|U(y, \mathrm{t})|\right]^{q} t^{-n} d y \frac{d t}{t}\right)^{p_{*} / q} d x\right\}^{1 / p_{*}} \\
& \lesssim\left\{\sup _{P \in Q} \frac{1}{|P|^{\tau}}\left(\int_{P}\left[G_{\lambda, r}^{0}(u)(x)\right]^{p} d x\right)^{1 / p}\right\}^{p / p_{*}}\left\|G_{\lambda, r}^{0}(u)\right\|_{L_{\tau}^{p}\left(\mathbb{R}^{n}\right)}^{1-\left(p / p_{*}\right)} \\
& \lesssim\left\|G_{\lambda, r}^{0}(u)\right\|_{L_{\tau}^{p}\left(\mathbb{R}^{n}\right)} \sim\|f\|_{F_{p, r}^{0, \tau}\left(\mathbb{R}^{n}\right)^{.}}
\end{aligned}
$$

When $f \in \dot{F}_{p, r}^{0, \tau}\left(\mathbb{R}^{n}\right)$ with $r \in(0,2)$, the desired conclusion is a direct consequence of the case $r \in[2, \infty]$, together with the the embedding $\dot{F}_{p, r}^{0, \tau}\left(\mathbb{R}^{n}\right) \subset \dot{F}_{p, 2}^{0, \tau}\left(\mathbb{R}^{n}\right)$ (see [14, Proposition 3.1 (i)]).

Case $2(\tau \in[1 / p, \infty))$. In this case, since $p_{*}>p$, we see that $\tau \geq 1 / p>1 / p_{*}$.

If $\tau \in(1 / p, \infty)$, by the assumption that $\ell>n / 2$, we know that there exists $\lambda>0$ such that $\ell>\lambda+n / 2$. Then from Remark 1.2, Theorem 2.3, Lemma 3.5(i) and the fact that $\dot{F}_{\infty, \infty}^{s}\left(\mathbb{R}^{n}\right)=\dot{B}_{\infty, \infty}^{s}\left(\mathbb{R}^{n}\right)$, it follows that

$$
\begin{aligned}
\left\|T_{m} f\right\|_{\dot{F}_{p *, q}^{\beta, \tau}\left(\mathbb{R}^{n}\right)} & \sim\left\|T_{m} f\right\|_{\dot{B}_{\infty, \infty}^{\beta+n\left(\tau-1 / p_{*}\right)}\left(\mathbb{R}^{n}\right)} \sim \sup _{t>0} \sup _{x \in \mathbb{R}^{n}} t^{-\beta-n\left(\tau-\left(1 / p_{*}\right)\right)}\left|T_{m} f * \Phi_{t}(x)\right| \\
& \lesssim \sup _{t>0} \sup _{x \in \mathbb{R}^{n}} t^{\alpha-\beta-n\left(\tau-\left(1 / p_{*}\right)\right)}\left|u_{\lambda}^{*}(x, t)\right| \sim \sup _{t>0} \sup _{x \in \mathbb{R}^{n}} t^{-n(\tau-(1 / p))}\left|u_{\lambda}^{*}(x, t)\right| \\
& \sim\|f\|_{\dot{B}_{\infty, \infty}^{n(\tau-1 / p)}\left(\mathbb{R}^{n)}\right)} \sim\|f\|_{\dot{F}_{p, r}^{0, \tau}\left(\mathbb{R}^{n}\right)} .
\end{aligned}
$$

If $\tau=1 / p$, we only consider the case $r=\infty$ in view of the embedding $\dot{F}_{p, r}^{0, \tau}\left(\mathbb{R}^{n}\right) \subset \dot{F}_{p, \infty}^{0, \tau}\left(\mathbb{R}^{n}\right)$ (see [14, Proposition 3.1(i)]). Then, similar to the above argument, we see that

$$
\left\|T_{m} f\right\|_{\hat{F}_{p *, q}^{\beta, \tau}} \sim \sup _{t>0} \sup _{x \in \mathbb{R}^{n}} t^{-\beta-n\left(\tau-1 / p_{*}\right)}\left|T_{m} f * \Phi_{t}(x)\right| \lesssim\|f\|_{\dot{F}_{\infty, \infty}^{0}\left(\mathbb{R}^{n}\right)} \sim\|f\|_{\dot{F}_{p, \infty}^{0, \tau}\left(\mathbb{R}^{n}\right)^{\prime}}
$$

which completes the proof of Theorem 1.7.

Now we give the proof of Corollary 1.8 .

Proof of Corollary 1.8. The result follows from either a minor modification of the proof of Theorem 1.7 or considering the symbols $\tilde{m}(\xi):=m(\xi)|\xi|^{-\gamma}$ for all $\xi \in \mathbb{R}^{n} \backslash\{0\}$ and the lifting property. We omit the details.

Next, we give the proof of Theorem 1.10 .

Proof of Theorem 1.10. Let $\Phi$ be as in Lemma 3.5.

(i) Since $\ell>n[\max (1 / p, 1 / r)+\tau+1 / 2]$, there exists $\lambda>n[\max (1 / p, 1 / r)+\tau]$ such that $\ell>\lambda+n / 2$. Then by Lemma 3.5(i), we see that for all $x \in \mathbb{R}^{n}$ and $t \in(0, \infty)$, (3.27) holds, which yields the desired result in view of Theorem 2.4. 
(ii) Since $\ell>n(1 / p+\tau+1 / 2)$, there exists $\lambda>n(1 / p+\tau)$ such that $\ell>\lambda+n / 2$. Then by Lemma 3.5(i), we also see that for all $x \in \mathbb{R}^{n}$ and $t \in(0, \infty)$, (3.27) holds, which yields the desired result in view of Theorem 2.5 and hence completes the proof of Theorem 1.10 .

Now we give the proof of Theorem 1.12. We begin with a technical lemma proved in [15, Lemma 3.2], which reflects the geometrical properties of Hausdorff capacities.

Lemma 3.6. Let $\beta \in[1, \infty), \lambda \in(0, \infty)$, and $\omega$ be a nonnegative Borel measurable function on $\mathbb{R}_{+}^{n+1}$. Then there exists a positive constant $C$, independent of $\beta, \omega$, and $\lambda$, such that

$$
H^{d}\left(\left\{x \in \mathbb{R}^{n}: N_{\beta} \omega(x)>\lambda\right\}\right) \leq C \beta^{d} H^{d}\left(\left\{x \in \mathbb{R}^{n}: N \omega(x)>\lambda\right\}\right),
$$

where $N_{\beta} \omega(x):=\sup _{|y-x|<\beta t} \omega(y, t)$ for all $x \in \mathbb{R}^{n}$.

Proof of Theorem 1.12. Since when $\tau=0$, the Triebel-Lizorkin-Hausdorff space is just the Triebel-Lizorkin space, we only give the proof for the case $\tau \in\left(0, \min \left\{1 /(p \vee r)^{\prime}, 1 /\left(p_{*} \vee q\right)^{\prime}\right\}\right]$.

Assume first that $f \in \mathcal{S}_{\infty}\left(\mathbb{R}^{n}\right)$ and $\|f\|_{F \dot{H}_{p, r}^{0, \tau}\left(\mathbb{R}^{n}\right)}>0$. Choose $\lambda>n / r$ and $\tilde{\omega}$ be a nonnegative function on $\mathbb{R}_{+}^{n+1}$ with

$$
\int_{\mathbb{R}^{n}}[N \tilde{\omega}(x)]^{(p \vee r)^{\prime}} d H^{n \tau(p \vee r)^{\prime}}(x) \leq 1
$$

such that

$$
\|f\|_{F \dot{H}_{p, r}^{0, \tau}\left(\mathbb{R}^{n}\right)} \leq\left\|G_{\lambda, r}^{0}(\tilde{\omega}, u)\right\|_{L^{p}\left(\mathbb{R}^{n}\right)} \leq 2\|f\|_{F \dot{H}_{p, r}^{0, \tau}\left(\mathbb{R}^{n}\right)}
$$

Then $\left\|G_{\lambda, r}^{0}(\tilde{\omega}, u)\right\|_{L^{p}\left(\mathbb{R}^{n}\right)}>0$.

Let $\varphi$ be as in (2.1). Then there exists a Schwartz function $\zeta$ such that $\widehat{\zeta}$ has compact support away from the origin and

$$
\int_{0}^{\infty} \widehat{\varphi}(s \xi) \widehat{\zeta}(s \xi) \frac{d s}{s}=1, \quad \xi \neq 0
$$

see, for example, [30,37]. By the Calderón reproducing formula, we know that for all $y \in \mathbb{R}^{n}$,

$$
\left(T_{m} f * \psi_{t}\right)(y)=\int_{0}^{\infty}\left(f * \varphi_{s} * K * \zeta_{s} * \psi_{t}\right)(y) \frac{d s}{s} .
$$

Then, applying Hölder's inequality, we conclude that for all nonnegative functions $\omega$ on $\mathbb{R}_{+}^{n+1}$ and $x \in B(y, t)$,

$$
\begin{aligned}
& \left|\left(T_{m} f * \psi_{t}\right)(y)\right|[\omega(y, t)]^{-1} \\
& \quad \lesssim G_{\lambda, r}^{0}(\tilde{\omega}, u)(x)\left\{\int_{0}^{\infty} \int_{\mathbb{R}^{n}}\left(1+\frac{|z-x|}{s}\right)^{\lambda r^{\prime}}\right.
\end{aligned}
$$


Journal of Function Spaces and Applications

$$
\begin{aligned}
& \left.\times\left|\left(K * \zeta_{s} * \psi_{t}\right)(y-z)\right|^{r^{\prime}}\left[\tilde{\omega}(z, s) \omega(y, t)^{-1}\right]^{r^{\prime}} s^{n\left(r^{\prime}-1\right)} d z \frac{d s}{s}\right\}^{1 / r^{\prime}} \\
& \lesssim G_{\lambda, r}^{0}(\tilde{\omega}, u)(x)\left\{\int_{0}^{\infty} \int_{\mathbb{R}^{n}}\left(1+\frac{|z-y|}{s}\right)^{\lambda r^{\prime}}\left(1+\frac{t}{s}\right)^{\lambda r^{\prime}}\right. \\
& \left.\times\left|\left(K * \zeta_{s} * \psi_{t}\right)(y-z)\right|^{r^{\prime}}\left[\tilde{\omega}(z, s) \omega(y, t)^{-1}\right]^{r^{\prime}} s^{n\left(r^{\prime}-1\right)} d z \frac{d s}{s}\right\}^{1 / r^{\prime}} \\
& =: G_{\lambda, r}^{0}(\tilde{\omega}, u)(x) h(y, t) .
\end{aligned}
$$

Raising this inequality to the power $p$ and integrating over the ball $B(y, t)$ with respect to $x$, we see that

$$
\left|\left(T_{m} f * \psi_{t}\right)(y)\right|[\omega(y, t)]^{-1} \lesssim\left\|G_{\lambda, q}^{0}(\tilde{\omega}, u)\right\|_{L^{p}\left(\mathbb{R}^{n}\right)} t^{-n / p} h(y, t)
$$

Since $\|f\|_{F \dot{H}_{p, r}^{0, \tau}}>0$, then $\left\|G_{\lambda, r}^{0}(\tilde{\omega}, u)\right\|_{L^{p}\left(\mathbb{R}^{n}\right)}>0$. Thus, in this case, for any fixed $x$ and $D:=D(x) \in(0, \infty)$ which is determined later, applying (3.43), (3.44), and the Aoki-Rolewicz theorem (see $[35,36])$, we know that

$$
\begin{aligned}
& \left\|T_{m} f\right\|_{F \dot{H}_{p *, q}^{\beta, \tau}\left(\mathbb{R}^{n}\right)}^{v} \\
& =\inf _{\omega}\left\|\left\{\left(\int_{0}^{D}+\int_{D}^{\infty}\right) \int_{|y-\cdot|<t} t^{-\beta q}\left|\left(T_{m} f * \psi_{t}\right)(y)\right|^{q}[\omega(y, t)]^{-q} \frac{d y d t}{t^{n+1}}\right\}^{1 / q}\right\|_{L^{p_{*}\left(\mathbb{R}^{n}\right)}}^{v} \\
& \lesssim \inf _{\omega} \|\left\{\int_{0}^{D} t^{-\beta q} \int_{|y-|<t}\left[G_{\lambda, r}^{0}(\tilde{\omega}, u) h(y, t)\right]^{q} d y \frac{d t}{t^{n+1}}\right. \\
& \left.+\int_{D}^{\infty} t^{-(\beta+(n / p)) q} \int_{|y-\cdot|<t}\left[\left\|G_{\lambda, r}^{0}(\tilde{\omega}, u)\right\|_{L^{p}\left(\mathbb{R}^{n}\right)} h(y, t)\right]^{q} d y \frac{d t}{t^{n+1}}\right\}^{1 / q} \|_{L^{p_{*}}\left(\mathbb{R}^{n}\right)}^{v} \\
& \lesssim \inf _{\omega} \|\left\{\int _ { 0 } ^ { D } t ^ { - \beta q } \int _ { | y - | < t } \left[\sum_{j}^{\infty} \sum_{i \in \mathbb{Z}} \int_{s \sim 2^{i t}} \int_{|z-y| \sim^{2} j_{s}}\left(1+\frac{|z-y|}{s}\right)^{\lambda r^{\prime}}\left(1+\frac{t}{s}\right)^{\lambda r^{\prime}}\right.\right. \\
& \times\left|\left(K * \zeta_{s} * \psi_{t}\right)(y-z)\right|^{r^{\prime}}\left[\tilde{\omega}(z, s) \omega(y, t)^{-1}\right]^{r^{\prime}} \\
& \left.\times s^{n\left(r^{\prime}-1\right)} d z \frac{d s}{s}\right]^{q / r^{\prime}} d y \frac{d t}{t^{n+1}}\left[G_{\lambda, r}^{0}(\tilde{\omega}, u)\right]^{q}
\end{aligned}
$$




$$
\begin{gathered}
\left.+\int_{D}^{\infty} t^{-\beta q-(n / p) q} \int_{|y-\cdot|<t}[\cdots]^{q / r^{\prime}} d y \frac{d t}{t^{n+1}}\left\|G_{\lambda, r}^{0}(\tilde{\omega}, u)\right\|_{L^{p}\left(\mathbb{R}^{n}\right)}^{q}\right\}^{1 / q} \|_{L^{p_{*}}\left(\mathbb{R}^{n}\right)}^{v} \\
\lesssim \sum_{j=0}^{\infty} \sum_{i \in \mathbb{Z}} \inf \|\left\{\int _ { 0 } ^ { D } t ^ { - \beta q } \int _ { | y - \cdot | < t } \left[\int_{s \sim 2^{i} t} \int_{|z-y| \dot{\sim}^{j} s}\left(1+\frac{|z-y|}{s}\right)^{\lambda r^{\prime}}\left(1+\frac{t}{s}\right)^{\lambda r^{\prime}}\right.\right. \\
\times\left|\left(K * \zeta_{s} * \psi_{t}\right)(y-z)\right|^{r^{\prime}}\left[\tilde{\omega}(z, s) \omega(y, t)^{-1}\right]^{r^{\prime}} \\
\left.\times s^{n\left(r^{\prime}-1\right)} d z \frac{d s}{s}\right]^{q / r^{\prime}} d y \frac{d t}{t^{n+1}}\left[G_{\lambda, r}^{0}(\tilde{\omega}, u)\right]^{q} \\
\left.+\int_{D}^{\infty} t^{-\beta q-(n / p) q} \int_{|y-|<t}[\cdots]^{q / r^{\prime}} d y \frac{d t}{t^{n+1}}\left\|G_{\lambda, r}^{0}(\tilde{\omega}, u)\right\|_{L^{p}\left(\mathbb{R}^{n}\right)}^{q}\right\}^{1 / q} \|_{L^{p_{*}}\left(\mathbb{R}^{n}\right)}^{v},
\end{gathered}
$$

where $v$ is as in Remark 2.6, $|z-y| \dot{\sim} 2^{j} s$ means $2^{j-1} s \leq|z-y|<2^{j} s$ for $j \in \mathbb{N}$ and $0 \leq|z-y|<s$ for $j=0, s \sim 2^{i} t$ means $2^{i-1} t \leq s<2^{i} t$ for $i \in \mathbb{Z}$.

For $(y, t) \in \mathbb{R}^{n} \times(0, \infty)$, let

$$
\omega_{i, j}(y, t):=2^{-(i+j) n \tau} \sup \left\{\tilde{\omega}(\xi, \delta):|\xi-y|<2^{j+1} \delta, 2^{-i-1} \leq \frac{\delta}{t} \leq 2^{i+1}\right\}
$$

Then by Lemma 3.6 and Remark $1.3, \omega_{i, j}$ satisfies that

$$
\int_{\mathbb{R}^{n}}\left[N \omega_{i, j}(x)\right]^{\left(p_{*} \vee q\right)^{\prime}} d H^{n \tau\left(p_{*} \vee q\right)} \leq 1
$$

modulo a positive constant.

Observing that $\tau(p \vee r)^{\prime} \leq \tau\left(p_{*} \vee q\right)^{\prime}$ and $p<p_{*}$, we know that $r \geq p_{*}>p$. We now show the desired conclusion in two cases for $r$ and $p$.

Case $1(r \in[2, \infty)$ and $p \in(1, \infty))$. By (1.27), there exist $\lambda>n / r$ and $\mu>n(\tau+1 / 2-1 / r)$ such that $\ell>\lambda+\mu$. Since $r \in[2, \infty)$, then by Hölder's inequality and Lemma 3.2(ii), we have

$$
\begin{aligned}
& \int_{|z-y| \dot{\sim}^{j} j_{s}}\left(1+\frac{|z-y|}{s}\right)^{\lambda r^{\prime}}\left|\left(K * \zeta_{s} * \psi_{t}\right)(y-z)\right|^{r^{\prime}} d z \\
& =\int_{|z| \dot{2}^{\prime} j_{s}}\left(1+\frac{|z|}{S}\right)^{-\mu r^{\prime}}\left(1+\frac{|z|}{S}\right)^{(\lambda+\mu) r^{\prime}}\left|\left(K * \zeta_{s} * \psi_{t}\right)(z)\right|^{r^{\prime}} d z \\
& \leq\left[\int_{|z| \dot{2}^{\prime} j_{s}}\left(1+\frac{|z|}{S}\right)^{-\mu r^{\prime}\left(2 /\left(2-r^{\prime}\right)\right)} d z\right]^{\left(2-r^{\prime}\right) / 2}
\end{aligned}
$$


Journal of Function Spaces and Applications

$$
\begin{aligned}
& \times\left\{\int_{|z| \dot{2} j_{s}}\left(1+\frac{|z|}{s}\right)^{2(\lambda+\mu)}\left|\left(K * \zeta_{s} * \psi_{t}\right)(z)\right|^{2} d z\right\}^{r^{\prime} / 2} \\
& \quad \lesssim 2^{j\left(-\mu r^{\prime}+n-n / 2 r^{\prime}\right)} s^{n\left(1-r^{\prime} / 2\right)}(\min \{t, s\})^{((-n / 2)+\alpha) r^{\prime}} \min \left\{1,\left(\frac{t}{s}\right)^{k r^{\prime}}\right\}\left(1+\frac{t}{s}\right)^{-N r^{\prime}},
\end{aligned}
$$

where $k, N$ are arbitrary positive integers, which are determined later. Hence, choosing $k>$ $n(\tau+1 / 2)$ and $N>\lambda+|\alpha|$, we see that

$$
\begin{aligned}
& \int_{s \sim 2^{i} t} \int_{|z-y| \dot{2}^{j} j_{s}}\left(1+\frac{|z-y|}{s}\right)^{\lambda r^{\prime}}\left(1+\frac{t}{s}\right)^{\lambda r^{\prime}}\left|\left(K * \zeta_{s} * \psi_{t}\right)(y-z)\right|^{r^{\prime}} s^{n\left(r^{\prime}-1\right)} d z \frac{d s}{s} \\
& \quad \lesssim 2^{j\left(-\mu r^{\prime}+n-(n / 2) r^{\prime}\right)} \int_{s \sim 2^{i} t} s^{(n / 2) r^{\prime}}(\min \{t, s\})^{(-n / 2+\alpha) r^{\prime}} \min \left\{1,\left(\frac{t}{s}\right)^{k r^{\prime}}\right\}\left(1+\frac{t}{s}\right)^{(\lambda-N) r^{\prime}} \frac{d s}{s} \\
& \quad \lesssim t^{\alpha r^{\prime}} 2^{j\left(-\mu r^{\prime}+n-(n / 2) r^{\prime}\right)} \min \left\{2^{-i(k-n / 2) r^{\prime}}, 2^{-i(\lambda-N-\alpha) r^{\prime}}\right\} .
\end{aligned}
$$

Thus, by choosing $\omega:=\omega_{i, j}$, we conclude that

$$
\begin{aligned}
& \left\|T_{m} f\right\|_{F \dot{H}_{* \neq, q}^{\beta, \tau}\left(\mathbb{R}^{n}\right)}^{v} \\
& \lesssim \sum_{j=0}^{\infty} 2^{j\left(n \tau-\mu+\left(n / r^{\prime}\right)-n / 2\right) v}\left[\sum_{i=0}^{\infty} 2^{i(n \tau-k+(n / 2)) v}+\sum_{i=-\infty}^{-1} 2^{i(n \tau+\alpha-\lambda+N) v}\right] \\
& \quad \times\left\|\left\{D^{(\alpha-B) q}\left[G_{\lambda, r}^{0}(\tilde{\omega}, u)\right]^{q}+D^{(\alpha-B-(n / p)) q}\left\|G_{\lambda, r}^{0}(\tilde{\omega}, u)\right\|_{L^{p}\left(\mathbb{R}^{n}\right)}^{q}\right\}^{1 / q}\right\|_{L^{p_{*}\left(\mathbb{R}^{n}\right)}}^{v} .
\end{aligned}
$$

Take $D$ such that

$$
D^{-n / p}=\frac{G_{\lambda, r}^{0}(\tilde{\omega}, u)(x)}{\left\|G_{\lambda, r}^{0}(\tilde{\omega}, u)\right\|_{L^{p}\left(\mathbb{R}^{n}\right)}}
$$

We then see that

$$
\begin{aligned}
\left\|T_{m} f\right\|_{F \dot{H}_{p *, q}^{\beta, \tau}\left(\mathbb{R}^{n}\right)}^{v} & \lesssim \sum_{j=0}^{\infty} 2^{j\left(n \tau-\mu+n / r^{\prime}-n / 2\right) v}\left[\sum_{i=0}^{\infty} 2^{i(n \tau-k+n / 2) v}+\sum_{i=-\infty}^{-1} 2^{i(n \tau+\alpha-\lambda+N) v}\right]\left\|G_{\lambda, r}^{0}(\tilde{\omega}, u)\right\|_{L^{p}\left(\mathbb{R}^{n}\right)}^{v} \\
& \lesssim\left\|G_{\lambda, r}^{0}(\tilde{\omega}, u)\right\|_{L^{p}\left(\mathbb{R}^{n}\right)^{\prime}}^{v}
\end{aligned}
$$

which, together Theorem 2.9, implies that $\left\|T_{m} f\right\|_{F \dot{H}_{p_{*}, q}^{\beta, \tau}\left(\mathbb{R}^{n}\right)} \lesssim\|f\|_{F \dot{H}_{p, r}^{0, \tau}\left(\mathbb{R}^{n}\right)}$ for all $f \in \mathcal{S}_{\infty}\left(\mathbb{R}^{n}\right)$. 
Case $2(1<p<r<2)$. By the assumption that $\ell>n(1 / r+1 / 2)$, there exists $\lambda>n / r$ such that $\ell>\lambda+n / 2$. Then by Lemma 3.2(i), we see that

$$
\begin{aligned}
(1+ & \left.\frac{|z|}{s}\right)^{\curlywedge}\left|\left(K * \zeta_{s} * \psi_{t}\right)(z)\right| \\
& \leq \int_{\mathbb{R}^{n}}\left(1+\frac{|z|}{s}\right)^{\curlywedge}\left|\left(K * \psi_{t}\right)(z-y)\right|\left|\zeta_{s}(y)\right| d y \\
& \leq \int_{\mathbb{R}^{n}}\left(1+\frac{|z-y|}{s}\right)^{\curlywedge}\left|\left(K * \psi_{t}\right)(z-y)\right|\left(1+\frac{|y|}{s}\right)^{\curlywedge}\left|\zeta_{s}(y)\right| d y \\
& \leq s^{-n}\left\{\sup _{y \in \mathbb{R}^{n}}(1+|y|)^{\lambda}|\zeta(y)|\right\} \int_{\mathbb{R}^{n}}\left(1+\frac{|z-y|}{s}\right)^{\curlywedge}\left|\left(K * \psi_{t}\right)(z-y)\right| d y \\
& \lesssim s^{-n}\left(\max \left\{1, \frac{t}{s}\right\}\right)^{\curlywedge} \int_{\mathbb{R}^{n}}\left(1+\frac{|z-y|}{t}\right)^{\curlywedge}\left|\left(K * \psi_{t}\right)(z-y)\right| d y \\
& \lesssim s^{-n} t^{\alpha}\left(\max \left\{1, \frac{t}{s}\right\}\right)^{\curlywedge} .
\end{aligned}
$$

From $p<r$ and $\tau \in\left(0, \min \left\{1 /(p \vee r)^{\prime}, 1 /\left(p_{*} \vee q\right)^{\prime}\right\}\right]$, it follows that $\tau r^{\prime} \leq 1$. Thus, by $\ell>\lambda+n / 2$, there exists $\mu>n \tau r^{\prime} / 2$ such that $\ell>\lambda+\mu$, which, together with Lemma 3.2(ii), implies that

$$
\begin{aligned}
\int_{|z-y| \dot{\sim}^{\prime} j_{s}}\left(1+\frac{|z-y|}{s}\right)^{\lambda r^{\prime}}\left|\left(K * \zeta_{s} * \psi_{t}\right)(y-z)\right|^{r^{\prime}} d z \\
\lesssim 2^{-2 \mu j}\left[s^{-n} t^{\alpha}\left(\max \left\{1, \frac{t}{s}\right\}\right)^{\lambda}\right]^{r^{\prime}-2} \\
\quad \times \int_{|z-y| 2^{j} s}\left(1+\frac{|z-y|}{s}\right)^{2(\lambda+\mu)}\left|\left(K * \zeta_{s} * \psi_{t}\right)(y-z)\right|^{2} d z \\
\lesssim 2^{-2 \mu j} s^{-n\left(r^{\prime}-2\right)} t^{\alpha\left(r^{\prime}-2\right)}\left(\max \left\{1, \frac{t}{s}\right\}\right)^{\lambda\left(r^{\prime}-2\right)}(\min \{t, s\})^{-n+2 \alpha} \\
\quad \times \min \left\{1,\left(\frac{t}{s}\right)^{2 k}\right\}\left(1+\frac{t}{s}\right)^{-2 N} .
\end{aligned}
$$


Then, choosing $k>n \tau r^{\prime} / 2+n / 2$ and $N>\left(\lambda r^{\prime} / 2\right)+|\alpha|$, we see that

$$
\begin{aligned}
\int_{s \sim 2^{i} t} & \int_{|z-y| \dot{2} 2^{j} s}\left(1+\frac{|z-y|}{s}\right)^{\lambda r^{\prime}}\left(1+\frac{t}{s}\right)^{\lambda r^{\prime}}\left|\left(K * \zeta_{s} * \psi_{t}\right)(y-z)\right|^{r^{\prime}} s^{n\left(r^{\prime}-1\right)} d z \frac{d s}{s} \\
\lesssim & 2^{-2 \mu j} t^{\alpha\left(r^{\prime}-2\right)} \int_{s \sim 2^{i} t} s^{n}\left(\max \left\{1, \frac{t}{s}\right\}\right)^{\lambda\left(r^{\prime}-2\right)}(\min \{t, s\})^{-n+2 \alpha} \\
& \times \min \left\{1,\left(\frac{t}{s}\right)^{2 k}\right\}\left(1+\frac{t}{s}\right)^{-2 N+\lambda r^{\prime}} \frac{d s}{s} \\
\lesssim & 2^{-2 \mu j} t^{\alpha r^{\prime}} \min \left\{2^{-i(2 k-n)}, 2^{2 i(\alpha+\lambda+N)}\right\} .
\end{aligned}
$$

This, together with the fact that $\mu>n \tau r^{\prime} / 2$ and an argument similar to Case 1 , further implies that for all $f \in \mathcal{S}_{\infty}\left(\mathbb{R}^{n}\right)$,

$$
\begin{aligned}
\left\|T_{m} f\right\|_{F \dot{H}_{p, q}^{\beta, \tau}\left(\mathbb{R}^{n}\right)}^{v,} & \lesssim \sum_{j=0}^{\infty} 2^{j\left(n \tau-2 \mu / r^{\prime}\right) v}\left[\sum_{i=0}^{\infty} 2^{i\left(n \tau-2 k / r^{\prime}+n / r^{\prime}\right) v}+\sum_{i=-\infty}^{-1} 2^{i\left(n \tau+2(\alpha+\lambda+N) / r^{\prime}\right) v}\right]\left\|G_{\lambda, r}^{0}(\tilde{\omega}, u)\right\|_{L^{p}\left(\mathbb{R}^{n}\right)}^{v} \\
& \lesssim\|f\|_{F \dot{H}_{p, r}^{0, \tau}\left(\mathbb{R}^{n}\right)^{\prime}}^{v}
\end{aligned}
$$

namely, $\left\|T_{m} f\right\|_{F \dot{H}_{p *, q}^{\beta, \tau}\left(\mathbb{R}^{n}\right)} \lesssim\|f\|_{F \dot{H}_{p, \tau}^{0, \tau}\left(\mathbb{R}^{n}\right)}$.

Next we assume that $f \in \mathcal{S}_{\infty}\left(\mathbb{R}^{n}\right)$ and $\|f\|_{F \dot{H}_{, r}^{0, \tau}\left(\mathbb{R}^{n}\right)}=0$. Then, for any $\varepsilon \in(0, \infty)$, there exists a nonnegative function $\tilde{\omega}$ on $\mathbb{R}_{+}^{n+1}$ such that $0 \leq\left\|G_{\lambda, r}^{0}(\tilde{\omega}, u)\right\|_{L^{p}\left(\mathbb{R}^{n}\right)}<\varepsilon$. If $\left\|G_{\lambda, r}^{0}(\tilde{\omega}, u)\right\|_{L^{p}\left(\mathbb{R}^{n}\right)}=0$, then $G_{\lambda, r}^{0}(\tilde{\omega}, u)(x)=0$ for almost every $x \in \mathbb{R}^{n}$, which, together with an argument similar to (3.43), further implies that $\left\|T_{m} f\right\|_{F \dot{H}_{p *, q}^{\beta, \tau}\left(\mathbb{R}^{n}\right)}=0$. If $\left\|G_{\lambda, r}^{0}(\tilde{\omega}, u)\right\|_{L^{p}\left(\mathbb{R}^{n}\right)}$ is positive, repeating the previous argument, we see that

$$
\left\|T_{m} f\right\|_{F \dot{H}_{p * q}^{\beta, \tau}\left(\mathbb{R}^{n}\right)} \lesssim \varepsilon
$$

for any $\varepsilon \in(0, \infty)$, and hence $\left\|T_{m} f\right\|_{F \dot{H}_{p *, q}^{\beta, \tau}\left(\mathbb{R}^{n}\right)}=0$. Thus, in this case, we also have $\left\|T_{m} f\right\|_{F \dot{H}_{p *, q}^{\beta, \tau}\left(\mathbb{R}^{n}\right)} \lesssim\|f\|_{F \dot{H}_{p, r}^{0, \tau}\left(\mathbb{R}^{n}\right)}$.

Finally, by the fact that $\mathcal{S}_{\infty}\left(\mathbb{R}^{n}\right)$ is dense in $F \dot{H}_{p, r}^{0, \tau}\left(\mathbb{R}^{n}\right)$ (see [13, Lemma 5.3]), together with a density argument, we know that the inequality $\left\|T_{m} f\right\|_{F \dot{H}_{p, q}^{\beta, \tau},\left(\mathbb{R}^{n}\right)} \lesssim\|f\|_{F \dot{H}_{p, r}^{0, \tau}\left(\mathbb{R}^{n}\right)}$ is true for all $f \in F \dot{H}_{p, r}^{0, \tau}\left(\mathbb{R}^{n}\right)$, which completes the proof of Theorem 1.12.

\section{Applications to Sobolev Embeddings}

As an application of Theorems 1.7 and 1.12, we give new direct proofs for the following Sobolev embedding theorems (see also [14, Proposition 3.3] and [15, Proposition 2.2]). 
Theorem 4.1. Let $\alpha, \beta \in \mathbb{R}, \alpha>\beta, q, r \in(0, \infty], p \in(0, \infty)$, and $\tau \in[0, \infty)$. If $p_{*} \in(0, \infty)$ such that $\beta-n / p_{*}=\alpha-n / p$, then $\dot{F}_{p, r}^{\alpha, \tau}\left(\mathbb{R}^{n}\right) \hookrightarrow \dot{F}_{p_{*}, q}^{\beta, \tau}\left(\mathbb{R}^{n}\right)$.

Proof. If we take $m(\xi):=|\xi|^{-\alpha}$ for all $\xi \in \mathbb{R}^{n} \backslash\{0\}$ in Theorem 1.7 and then apply the lifting property (see [14, Proposition 3.5]), we immediately obtain the desired conclusion of Theorem 4.1, which completes the proof of Theorem 4.1.

Theorem 4.2. Let $\alpha, \beta \in \mathbb{R}, \alpha>\beta$, and $p \in(1, \infty)$. Assume that $p_{*} \in(1, \infty)$ satisfies $\beta-n / p_{*}=$ $\alpha-n / p$. Let $r, q \in(1, \infty)$, and $\tau \in\left[0, \min \left\{1 /\left(p_{*} \vee q\right)^{\prime}, 1 /(p \vee r)^{\prime}\right\}\right]$ such that $\tau(p \vee r)^{\prime} \leq \tau\left(p_{*} \vee q\right)^{\prime}$. Then $F \dot{H}_{p, r}^{\alpha, \tau}\left(\mathbb{R}^{n}\right) \hookrightarrow F \dot{H}_{p_{*}, q}^{\beta, \tau}\left(\mathbb{R}^{n}\right)$.

Proof. If we take $m(\xi):=|\xi|^{-\alpha}$ for all $\xi \in \mathbb{R}^{n} \backslash\{0\}$ in Theorem 1.12 and then apply the lifting property which can be deduced directly from [15, Theorem 4.1], we immediately obtain the desired conclusion of Theorem 4.2, which completes the proof of Theorem 4.2.

\section{Acknowledgments}

D. Yang would like to thank Professor Hans Triebel and Professor Winfried Sickel for some suggestive and helpful discussions on this paper. D. Yang is supported by the National Natural Science Foundation (Grant no. 11171027) of China and Program for Changjiang Scholars and Innovative Research Team in University of China. W. Yuan is supported by the National Natural Science Foundation (Grant no. 11101038) of China.

\section{References}

[1] S. G. Mihlin, "On the multipliers of Fourier integrals," Doklady Akademii Nauk SSSR, vol. 109, pp. 701-703, 1956 (Russian).

[2] S. G. Michlin, "Fourier integrals and multiple singular integrals," Vestnik, Leningrad University, vol. 12, no. 7, pp. 143-155, 1957 (Russian).

[3] L. Hörmander, "Estimates for translation invariant operators in $L^{p}$ spaces," Acta Mathematica, vol. 104, pp. 93-140, 1960.

[4] H. Triebel, "Spaces of distributions of Besov type on Euclidean n-space. Duality, interpolation," Arkiv för Matematik, vol. 11, pp. 13-64, 1973.

[5] H. Triebel, Interpolation Theory, Function Spaces, Differential Operators, Johann Ambrosius Barth, Heidelberg, Germany, 2nd edition, 1995.

[6] J. Peetre, New Thoughts on Besov Spaces, Mathematics Department, Duke University, Durham, NC, USA, 1976.

[7] D. G. Orlovski1, "On multipliers in the spaces $B_{p, \theta}^{r}{ }^{\prime}$ " Analysis Mathematica, vol. 5, no. 3, pp. 207-218, 1979.

[8] P. I. Lizorkin, "Bases and multipliers in the spaces $B_{r}^{p \theta}(\Pi)$, " in Proceedings of the Steklov Institute of Mathematics, vol. 143, pp. 93-110, 1980.

[9] H. Triebel, "Complex interpolation and Fourier multipliers for the spaces $B_{p, q}^{s}$ and $F_{p, q}^{s}$ of BesovHardy-Sobolev type: the case $0<p \leq \infty, 0<q \leq \infty$," Mathematische Zeitschrift, vol. 176, no. 4, pp. 495$510,1981$.

[10] H. Triebel, Theory of Function Spaces, Birkhäuser, Basel, Switzerland, 1983.

[11] Y.-K. Cho and D. Kim, "A Fourier multiplier theorem on the Besov-Lipschitz spaces," The Korean Mathematical Society, vol. 16, pp. 85-90, 2008.

[12] Y.-K. Cho, "Continuous characterization of the Triebel-Lizorkin spaces and Fourier multipliers," Bulletin of the Korean Mathematical Society, vol. 47, no. 4, pp. 839-857, 2010.

[13] D. Yang and W. Yuan, "A new class of function spaces connecting Triebel-Lizorkin spaces and $Q$ spaces," Journal of Functional Analysis, vol. 255, no. 10, pp. 2760-2809, 2008.

[14] D. Yang and W. Yuan, "New Besov-type spaces and Triebel-Lizorkin-type spaces including $Q$ spaces," Mathematische Zeitschrift, vol. 265, no. 2, pp. 451-480, 2010. 
[15] W. Yuan, Y. Sawano, and D. Yang, "Decompositions of Besov-Hausdorff and Triebel-Lizorkin-Hausdorff spaces and their applications," Journal of Mathematical Analysis and Applications, vol. 369, no. 2, pp. 736-757, 2010.

[16] Y. Sawano, D. Yang, and W. Yuan, "New applications of Besov-type and Triebel-Lizorkin-type spaces," Journal of Mathematical Analysis and Applications, vol. 363, no. 1, pp. 73-85, 2010.

[17] W. Yuan, W. Sickel, and D. Yang, Morrey and Campanato meet Besov, Lizorkin and Triebel, vol. 2005 of Lecture Notes in Mathematics, Springer, Berlin, Germany, 2010.

[18] Y. Liang, Y. Sawano, T. Ullrich, D. Yang, and W. Yuan, "New characterizations of Besov-TriebelLizorkin-Hausdorff spaces including coorbits and wavelets," Mathematics. In press. http:/ /arxiv.org/ abs/1103.5667/.

[19] H. Triebel, Theory of Function Spaces. II, Birkhäuser, Basel, Switzerland, 1992.

[20] H. Triebel, Theory of Function Spaces. III, Birkhäuser, Basel, Switzerland, 2006.

[21] M. Essén, S. Janson, L. Peng, and J. Xiao, "Q spaces of several real variables," Indiana University Mathematics Journal, vol. 49, no. 2, pp. 575-615, 2000.

[22] G. Dafni and J. Xiao, "Some new tent spaces and duality theorems for fractional Carleson measures and $Q_{\alpha}\left(\mathbb{R}^{n}\right)$," Journal of Functional Analysis, vol. 208, no. 2, pp. 377-422, 2004.

[23] J. Xiao, Holomorphic Q Classes, vol. 1767 of Lecture Notes in Mathematics, Springer, Berlin, Germany, 2001.

[24] J. Xiao, Geometric $Q_{p}$ Functions, Birkhäuser, Basel, Switzerland, 2006.

[25] L. Tang and J. Xu, "Some properties of Morrey type Besov-Triebel spaces," Mathematische Nachrichten, vol. 278, no. 7-8, pp. 904-917, 2005.

[26] Y. Sawano and H. Tanaka, "Decompositions of Besov-Morrey spaces and Triebel-Lizorkin-Morrey spaces," Mathematische Zeitschrift, vol. 257, no. 4, pp. 871-905, 2007.

[27] Y. Sawano, "Wavelet characterization of Besov-Morrey and Triebel-Lizorkin-Morrey spaces," Approximatio Commentarii Mathematici, vol. 38, pp. 93-107, 2008.

[28] Y. Sawano, "A note on Besov-Morrey spaces and Triebel-Lizorkin-Morrey spaces," Acta Mathematica Sinica, vol. 25, no. 8, pp. 1223-1242, 2009.

[29] D. Yang and W. Yuan, "Relations among Besov-type spaces, Triebel-Lizorkin-type spaces and generalized Carleson measure spaces," Applicable Analysis. In press.

[30] M. Frazier and B. Jawerth, "A discrete transform and decompositions of distribution spaces," Journal of Functional Analysis, vol. 93, no. 1, pp. 34-170, 1990.

[31] D. R. Adams, "A note on Choquet integrals with respect to Hausdorff capacity," in Function Spaces and Applications, vol. 1302 of Lecture Notes in Mathematics, pp. 115-124, Springer, Berlin, Germany, 1988.

[32] D. Yang and W. Yuan, "A note on dyadic Hausdorff capacities," Bulletin des Sciences Mathématiques, vol. 132, no. 6, pp. 500-509, 2008.

[33] E. M. Stein, Singular Integrals and Differentiability Properties of Functions, vol. 30 of Princeton Mathematical Series, Princeton University Press, Princeton, NJ, USA, 1970.

[34] J. Marschall, "Nonregular pseudo-differential operators," Zeitschrift für Analysis und ihre Anwendungen, vol. 15, no. 1, pp. 109-148, 1996.

[35] T. Aoki, "Locally bounded linear topological spaces," Proceedings of the Imperial Academy, vol. 18, pp. 588-594, 1942.

[36] S. Rolewicz, "On a certain class of linear metric spaces," Bulletin of the Polish Academy of Sciences, vol. 5, pp. 471-473, 1957.

[37] M. Frazier, B. Jawerth, and G. Weiss, "Littlewood-Paley theory and the study of function spaces," in Proceedings of the Conference Board of the Mathematical Sciences, vol. 79 of CBMS Regional Conference Series in Mathematics, the American Mathematical Society, Washington, DC, USA, 1991. 


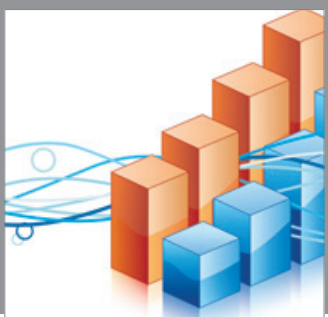

Advances in

Operations Research

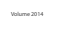

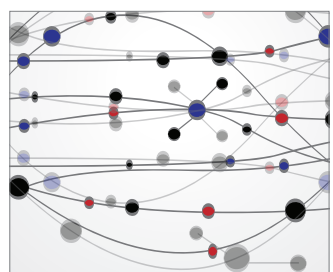

\section{The Scientific} World Journal
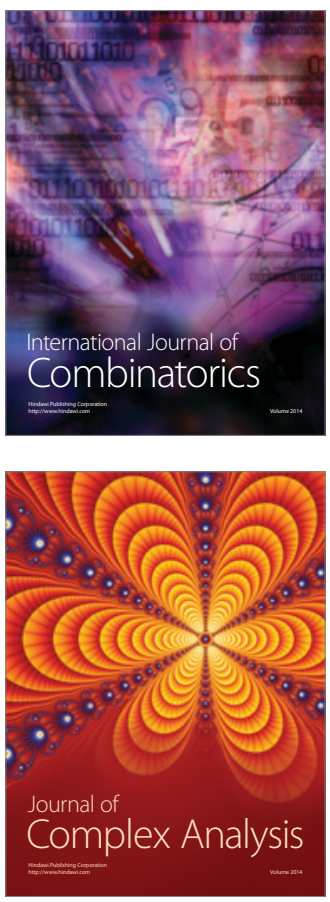

International Journal of

Mathematics and

Mathematical

Sciences
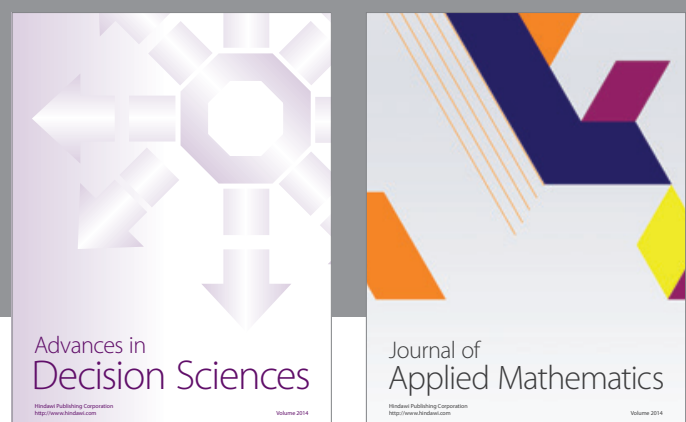

Journal of

Applied Mathematics
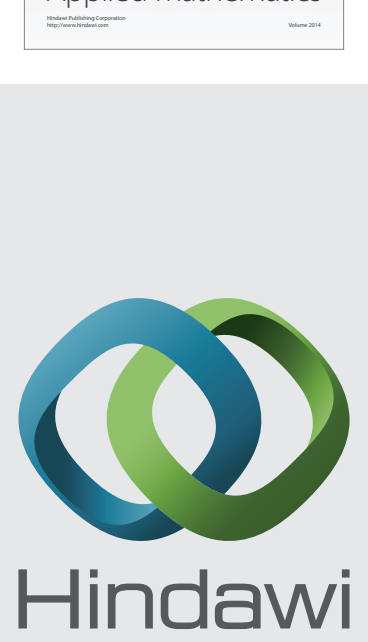

Submit your manuscripts at http://www.hindawi.com
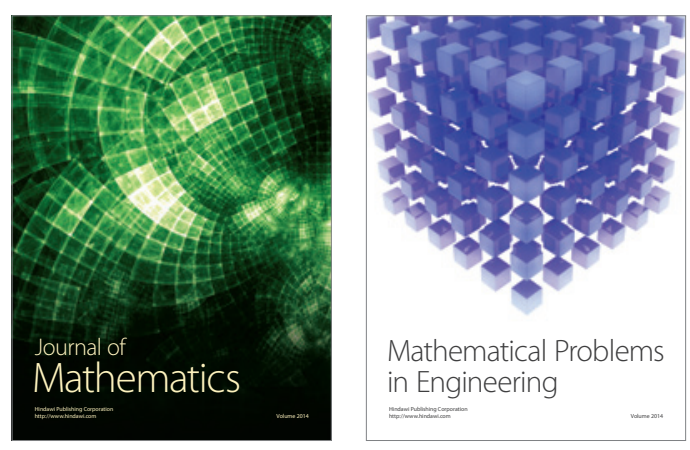

Mathematical Problems in Engineering
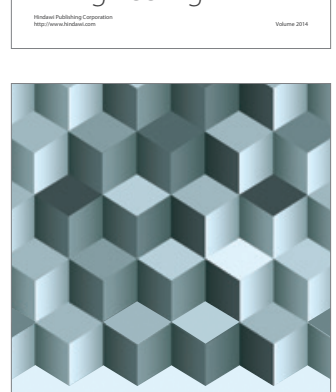

Journal of

Function Spaces
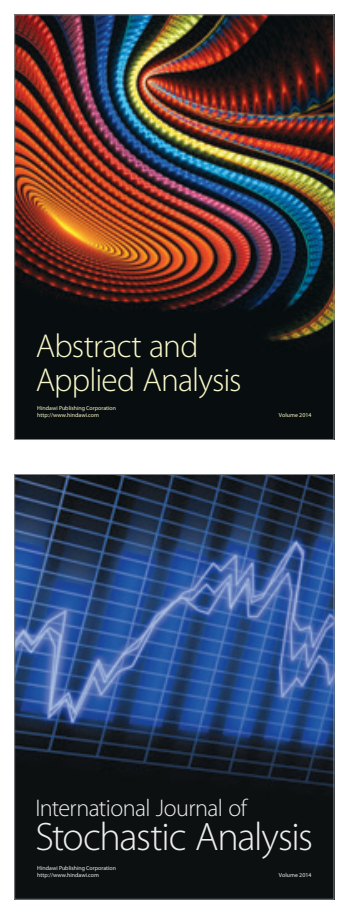

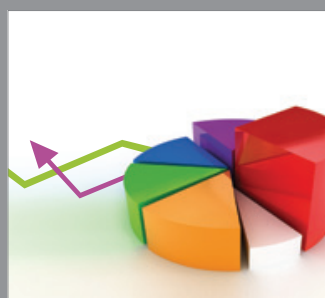

ournal of

Probability and Statistics

Promensencen
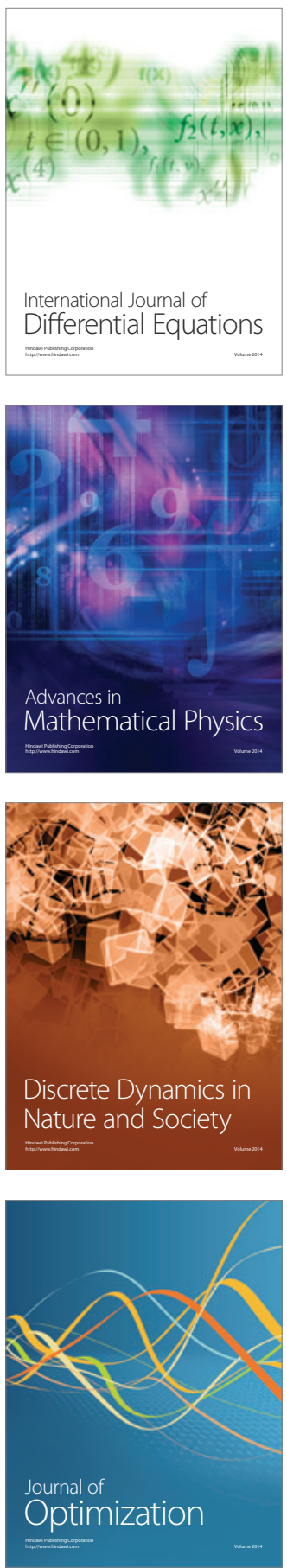